\title{
PETROLOGY AND GEOCHEMISTRY OF WADI ATALLA EL-MURR METABASALTS, EASTERN DESERT, EGYPT: VOLCANISM IN A MATURE NEOPROTEROZOIC BACK-ARC BASIN
}

\author{
Ayman E. Maurice \\ Geology Department, Faculty of Science, Helwan University, 1795 Cairo, Egypt
}

\begin{abstract}
The mafic volcanic rocks of Wadi Atalla El-Murr, Eastern Desert, Egypt, comprise aphyric and porphyritic metabasalts composed of variably altered pyroxene and plagioclase. In the porphyritic metabasalts, the phenocrysts are dominated by augite either as individual crystals or as aggregates forming glomeroporphyritic texture. The plagioclases encompass a wide compositional range from labradorite (An $=\sim 73 \%)$ to albite $(\mathrm{An}=0.61 \%)$, reflecting seafloor hydrothermal alteration and metamorphism. The composition of the augite indicates crystallization from a subalkaline magma, overlapping the chemical characteristics of pyroxenes from island arc and ocean-floor basalts. The whole-rock compositions of representative samples reveal that these basalts are characterized by low $\mathrm{K}_{2} \mathrm{O}$ contents $(<1 \mathrm{wt} \%)$ and have tholeiitic affinity. The chondrite-normalized REE patterns of the metabasalts display slight enrichment in LREE relative to HREE $\left(\mathrm{La} / \mathrm{Yb}_{\mathrm{n}}=1.08-1.70\right)$ and their MORB-normalized spider diagrams show variable LILE enrichment, variable depletion in most HFSE and a small negative $\mathrm{Nb}$ anomaly, suggesting derivation from a mantle source modified by a subduction component. However, these tholeiitic basalts differ in their geochemical features from Neoproterozoic nascent intra-oceanic island arc tholeiites (IAT), and mostly occupy the MORB or BABB field on conventional tectonomagmatic discrimination diagrams. The mixed MORB/IAT geochemical characteristics of the Wadi Atalla El-Murr basalts most closely resemble those of basalts generated in modern back-arc environments affected by minor subduction input, implying eruption during the earliest mature stage of a Neoproterozoic back-arc basin. The present and published data of the metavolcanic rocks of Egyptian ophiolites revealed that they comprise BABB and MORB compositions. The majority of ophiolite metavolcanics of Egypt formed in subduction-related settings, from mantle sources slightly to significantly modified by input of a subduction component, implying generation at different stages of development of Neoproterozoic supra-subduction zone systems.
\end{abstract}

Keywords: Neoproterozoic ophiolites, Ophiolite settings, Back-arc basins, BABB, Eastern Desert

\section{INTRODUCTION}

Oceanic crust has been essentially created along mid-oceanic ridges or smaller back-arc spreading centers (Wilson, 1989), however, the erupted volcanics in these settings may differ geochemically. Volcanic rocks erupted along modern back-arcs, such as Lau basin (e.g. Pearce et al., 1995; Keller et al., 2008; Tian et al., 2011), Okinawa Trough (e.g. Shinjo et al., 1999), Japan Sea back-arc basin (e.g. Hirahara et al., 2015), Manus back-arc basin (Sinton et al., 2003), Mariana Trough (e.g. Gribble et al., 1998; Pearce et al., 2005) and East Scotia Sea (e.g. Tarney et al., 1981; Fretzdorff et al., 2002), show geochemical variations from typical MORB to typical arc volcanics (e.g. Fretzdorff et al., 2002; Pearce et al., 1995; Keller et al., 2008). The variable arc signature in back-arc lavas is related to the proximity of spreading center from volcanic arc (e.g. Tarney et al., 1981; Pearce et al., 1995) and to the amount and type (fluid or melt) of subduction component (e.g. Pearce et al., 1995; Hirahara et al., 2015). Although back-arc lavas are essentially basaltic in composition, some back-arc basins erupts more evolved volcanics (e.g. Vallier et al., 1991; Pearce et al., 1995; Tian et al., 2011).

Neoproterozoic intra-oceanic volcanics of the Eastern Desert of Egypt comprise ophiolite (e.g. Abd ElRahman et al., 2009a\&b; Basta et al., 2011) and island arc (e.g. Maurice et al., 2012) metavolcanics. The ophiolite metavolcanics are essentially basalts, basaltic andesites and andesites with tholeiitic to 
Maurice, A. E.

transitional character (e.g. Abd El-Rahman et al., 2009a; Basta et al., 2011) whereas the island arc metavolcanics range from tholeiitic basalts (e.g. Maurice et al., 2012) to calc-alkaline andesites (e.g. Stern et al., 1981). Although the ophiolite and island arc metavolcanics are termed, respectively, older and younger metavolcanics (Stern et al., 1981), U-Pb zircon dating of these volcanics revealed that they have similar ages ( 750 Ma, Ali et al., 2009). The ophiolite metavolcanics are considered to be generated in a major ocean basin (e.g. Zimmer et al., 1995), back-arc (e.g. El-Sayed et al., 1999; Abd El-Rahman et al., 2009a; Basta et al., 2011) or incipient fore-arc (Abd El-Rahman et al., 2009b). On the other hand, the arc tholeiitic basalts and calc-alkaline andesites were formed during nascent (Maurice et al., 2012) and mature stages (Stern et al., 1981), respectively, of intra-oceanic island arcs. Recently, Abdel-Karim et al. (2019) proposed that the tholeiitic/calc-alkaline Shadli bimodal metavolcanics were erupted in arc setting. The third class of the Neoproterozoic volcanic rocks in the Eastern Desert of Egypt is represented essentially by calc-alkaline andesitic/dacitic Dokhan volcanics (e.g. Basta et al., 1980; Ressetar and Monrad; 1983; Abdel-Rahman, 1996; Moghazi, 2003; Eliwa et al., 2006). The tectonic setting and the origin of the Dokhan volcanics have been controversial (Maurice et al., 2018 and references therein).

This study present petrography, mineral chemistry and whole-rock geochemistry of Neoproterozoic metabasalts from Wadi Atalla El-Murr area, Central Eastern Desert, Egypt, aiming to decipher their tectonic setting. The nature of the Neoproterozoic mantle source of the parental magmas of these oceanic basalts and the subduction component contributed to their genesis have been evaluated. The implication of the results of the present study for classification of Eastern Desert ophiolite metavolcanics has been considered.

\section{GEOLOGIC SETTING}

The Wadi Atalla El-Murr area is part of Wadi Atalla district, Central Eastern Desert, Egypt. This area hosts two gold occurrences, namely Atalla El-Murr and El-Nabsh El-Kidim. The mapped area (Fig. 1) is covered by serpentinites, volcanic rocks, felsites, younger gabbros and younger granites (Essawy and Abu Zeid, 1972; Ghoneim, 1973; Wassef et al., 1973; Bakhit, 1994; Basta et al., 1996). The small sepentinites mass in the southwestern part of the mapped area constitutes part of mountainous ultramafic chain which extends NW-ward from Fawakhir area in the south. The volcanic rocks cover most of the study area and crop out essentially in the eastern side of Wadi Atalla. They were previously mapped as island arc volcanics (Bakhit, 1994; Basta et al., 1996), and comprise mafic and felsic varieties. The mafic volcanics are the dominant rocks, occupying most of the study area, while the felsic volcanics are exposed in the southeastern part. The gold occurrences are located within the mafic volcanics mass, which is dissected by numerous quartz veins. Taman (1996) studied volcanic rocks in part of Wadi Atalla El-Murr area and proposed that they comprise rhyolitic flows and tuffs which are cut by intermediate and mafic dykes, quartz veins and rare jasperoid veins. In the western part of the study area, the felsites are exposed as NWtrending rugged belt intruding the metavolcanics (Ghoneim, 1973; Bakhit, 1994; Basta et al., 1996). The younger gabbros were recorded by Bakhit (1994) as cone hills in the southern part of the study area. The younger granites are represented by a small pluton traversed by Wadi Atalla El-Murr in the northern part of the study area. They are intruding the metavolcanics and cut by dykes.

\section{PETROGRAPHY}

The metabasalts of Wadi Atalla El-Murr area are classified petrographically into non-porphyritic basalt, vitrophyre and porphyritic basalt, which is occasionally amygdaloidal. The variation of texture from very fine-grained through vitrophyre to porphyritic reflects variable degrees of cooling from quenching to slower rate of cooling. The opaque minerals are represented essentially by ilmenite which is mostly altered to titanite.

\section{Non-porphyritic basalt}

The rock is very fine- to fine-grained, composed of fine pyroxene and very fine to fine plagioclase laths (Fig. 2A). Sometimes, pyroxene is present as rare microphenocrysts. The pyroxenes are commonly altered to tremolite and chlorite. Carbonate and chlorite are secondary minerals. The rock is occasionally dissected by carbonates-quartz and quartz veinlets. 
Fig. 1: Location and geologic map of Wadi Atalla El-Murr area (modified after Wassef et al., 1973), Central Eastern Desert (CED), Egypt.

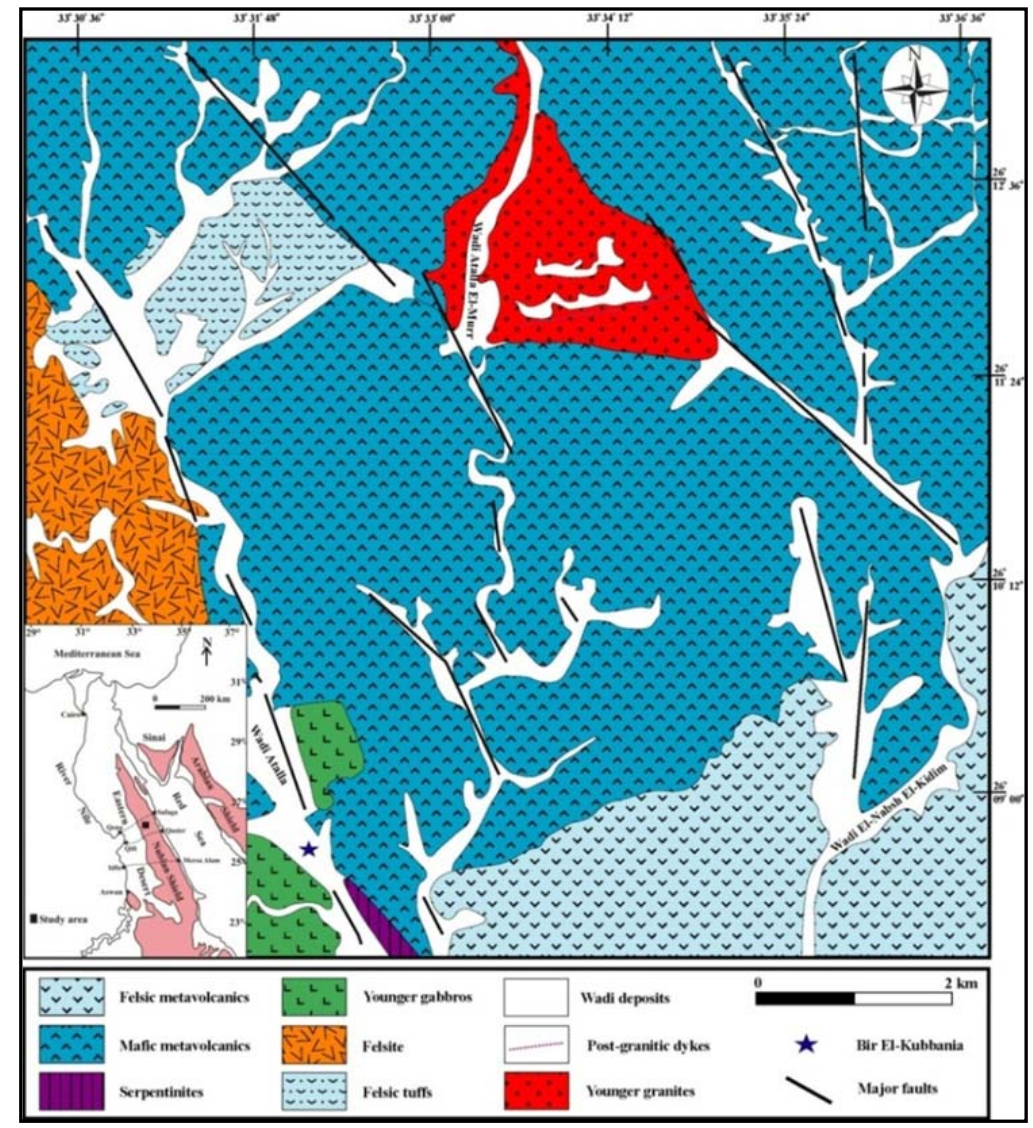

\section{Vitrophyre}

The rock is composed of pyroxene phenocrysts/microphenocrysts and plagioclase microphenocrysts within very fine-grained groundmass (Fig. 2B). The phenocrysts are mostly pyroxene, while the plagioclase microphenocrysts are subordinate and occur as thin prisms and laths. The very fine-grained groundmass is composed of devitrified glassy material. The pyroxene is partly altered to chlorite and plagioclase is slightly sericitized. The rock is dissected with chlorite and chlorite-quartz veins.

\section{Porphyritic basalt}

The rock is composed of pyroxene phenocrysts and occasionally rare plagioclase phenocrysts in finegrained groundmass (Fig. 2C). Pyroxene phenocrysts occur either as equant individual crystals or as clusters, forming glomeroporphyritic texture (Fig. 2D). Pyroxene is also present as finer prismatic crystals. The pyroxene is partly altered to tremolite and chlorite (Fig. 2E), and plagioclase is variably sericitized. The rock is sometimes amygdaloidal and displays quench texture (Fig. 2F). The amygdales are irregular in shape and filled with chlorite and/or calcite. The fine-grained groundmass is composed of fine prismatic pyroxene and plagioclase as well as devitrified glass. The rock is dissected by veinlets of chlorite, chlorite-epidote and carbonate with or without chlorite.

\section{ANALYTICAL TECHNIQUES}

Representative whole-rock samples of the different basalts were analyzed for major, trace and rare earth elements using wave-length dispersive X-ray fluorescence spectrometer (WD-XRF, Axios, PANalytical) and laser ablation - inductively coupled plasma - mass spectrometry (LA-ICP-MS). Pyroxene and plagioclase analyses were carried out using a JEOL JXA-8200 electron probe microanalyzer equipped with five wavelength dispersive spectrometers (WDS). The bulk-rock and electron microprobe analyses were performed at the Institute of Geochemistry and Petrology, ETH-Zürich, Switzerland. The description and detection limits of the methods used in whole-rock and minerals analyses are similar to those given in Basta et al. (2011, 2017). 
Maurice, A. E.
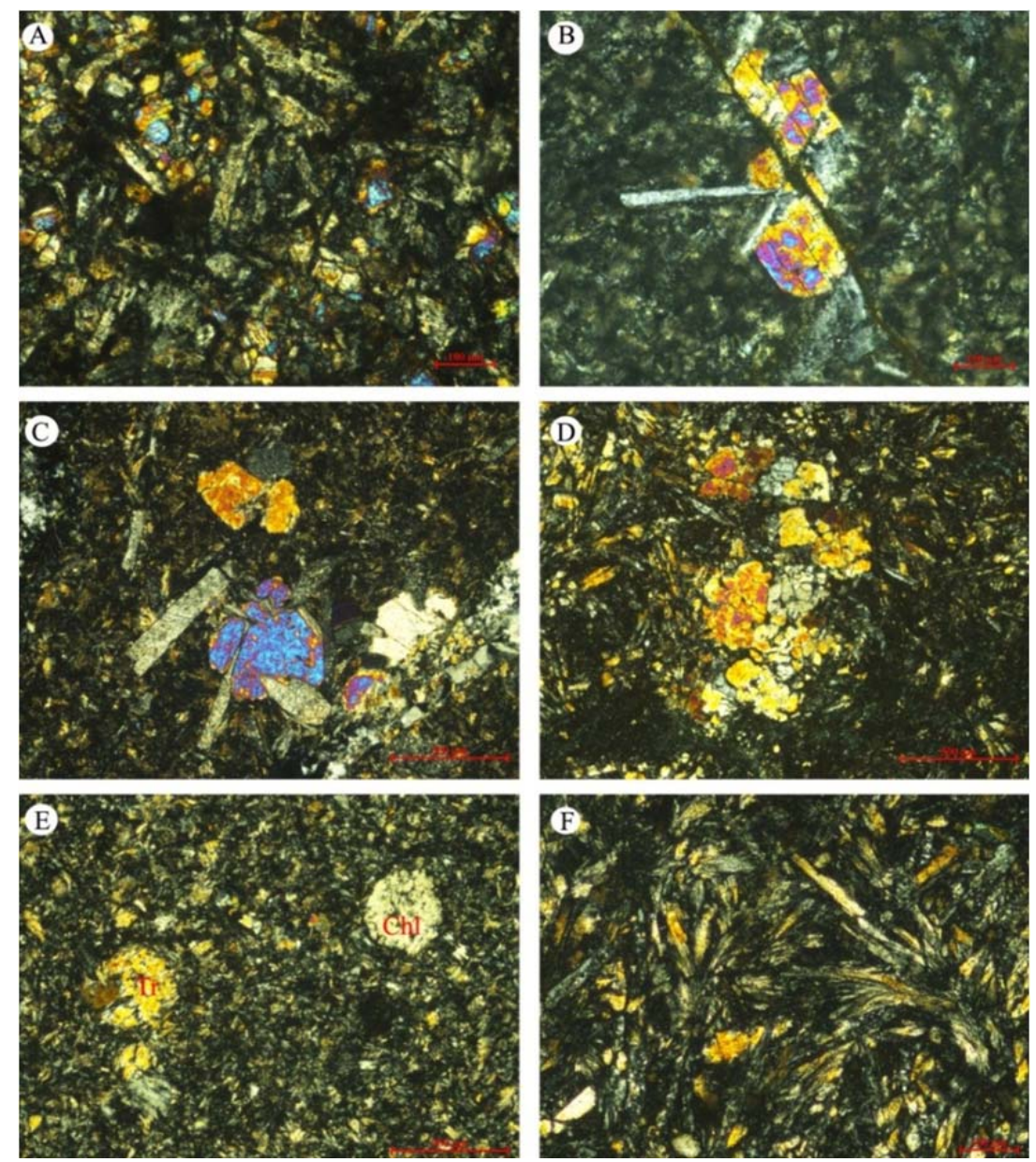

Fig. 2: Petrographic characteristics of Wadi Atalla El-Murr metabasalts. (A) Aphyric metabasalt composed of pyroxene and plagioclase, (B) Vitrophyre showing pyroxene phenocrysts and plagioclase microphenocrysts in devitrified groundmass, (C) Porphyritic basalt showing pyroxene and plagioclase phenocrysts/microphenocrysts, (D) Porphyritic basalt showing glomeroporphyritic texture, (E) Porphyritic basalt showing pyroxene phenocrysts altered to tremolite (Tr) and chlorite (Chl), (F) Quench texture in porphyritic basalt, reflecting rapid cooling. All photos are in crossed nicols.

\section{MINERAL CHEMISTRY}

Fresh relics of pyroxene and plagioclase of Wadi Atalla El-Murr metabasalts were analyzed by electron microprobe. The composition and structural formula of the analyzed pyroxene and plagioclase are given in Tables 1 and 2.

\section{Pyroxene}

The pyroxenes of Wadi Atalla El-Murr metabasalts belong to the calcic pyroxenes with $\mathrm{CaO}$ contents ranging from $15-21 \mathrm{wt} \%$ (average $=19 \mathrm{wt} \%$ ) and are classified as augites (Fig. 3A) following the

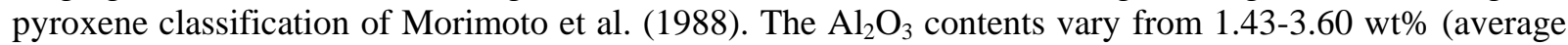
$=2.31 \mathrm{wt} \%)$. These pyroxenes are characterized by low $\mathrm{TiO}_{2}(0.25-0.75 \mathrm{wt} \%$, average $=0.40 \mathrm{wt} \%)$ and $\mathrm{Cr}_{2} \mathrm{O}_{3}$ (0-0.48 wt $\%$, average $\left.=0.14 \mathrm{wt} \%\right)$ contents and are similar to pyroxenes crystallize from subalkaline magmas (Fig. 3B). They have mineral chemical characteristics akin to those of pyroxenes of both island arc and ocean-floor basalts (Fig. 3C and D).

\section{Plagioclase}

The analyzed plagioclases of Wadi Atalla El-Murr metabasalts show wide range of $\mathrm{CaO}$ (0.11-14.45 wt $\%$, average $=5.53 \mathrm{wt} \%)$ and $\mathrm{Na}_{2} \mathrm{O}(2.90-9.89 \mathrm{wt} \%$, average $=7.27 \mathrm{wt} \%)$ contents. They cover wide 


\section{Petrology and geochemistry of Wadi Atalla El-Murr}

compositional range from labradorite to albite (Table 2). The high An contents (up to 73\%) of plagioclases indicate primary composition, whereas the low An content (down to 0.61\%) of some plagioclases reflects post-magmatic modifications. Such broad variation in the chemical composition of plagioclases and presence of albite in these basalts can be attributed to sea-floor hydrothermal alteration and metamorphism.

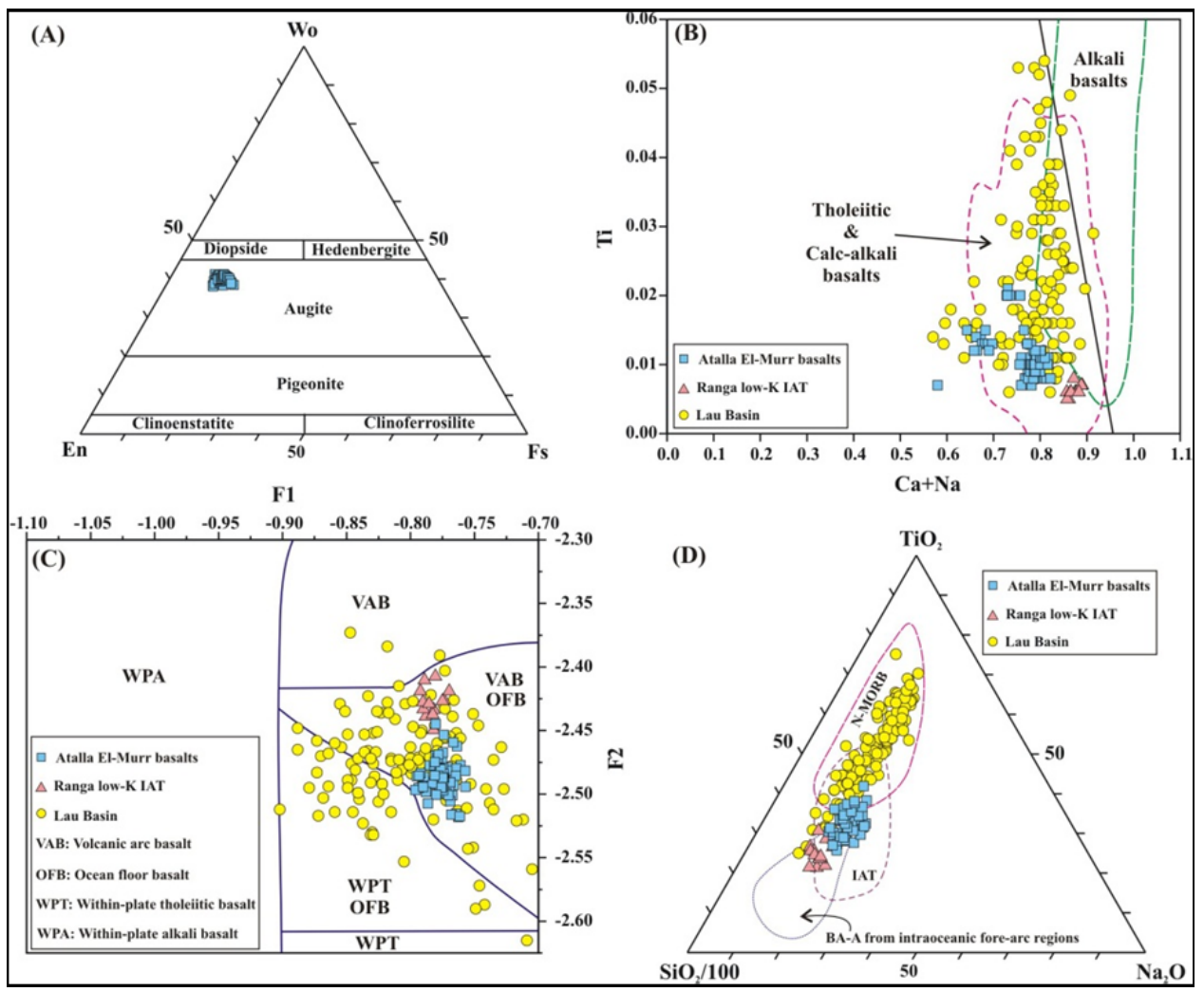

Fig. 3: Compositional features of the pyroxene of Wadi Atalla El-Murr basalts. (A) En-Fs-Wo diagram for nomenclature of pyroxene (after Morimoto et al., 1988), (B) $\mathrm{Ti}$ vs $\mathrm{Ca}+\mathrm{Na}$ diagram (after Leterrier et al., 1982) for evaluation of magma type, (C) F1-F2 diagram (after Nisbet and Pearce, 1977) and (D) $\mathrm{TiO}_{2^{-}}$ $\mathrm{Na}_{2} \mathrm{O}-\mathrm{SiO}_{2} / 100$ diagram (after Beccaluva et al., 1989) for tectonic setting discrimination. Data sources: pyroxene composition of Lau Basin back-arc basalts after Hawkins and Allan (1994); pyroxene composition of Neoproterozoic IAT of Wadi Ranga (South Eastern Desert) after Maurice et al. (2012).

\section{GEOCHEMISTRY}

Thirteen representative samples of Wadi Atalla El-Murr mafic volcanic rocks have been selected for major, trace and rare earth elements analyses using XRF and LA-ICP-MS techniques. The compositions of the analyzed whole-rock samples are given in Table 3.

The mafic volcanic rocks of Wadi Atalla El-Murr are classified as basalt (Fig. 4A). Moreover, the basalts plot in the field of the older metavolcanics (OMV) of Eastern Desert (Fig. 4A). The LOI values of all analyzed metabasalts (except one) are less than or equal to $4 \mathrm{wt} \%$ indicating that the magmatic composition of these rocks was not significantly modified by alteration and metamorphism (Polat et al., 2002). The metabasalts have low $\mathrm{K}_{2} \mathrm{O}$ contents (0.15-0.94 wt\%) similar to oceanic volcanic rocks derived from depleted mantle source (Maurice et al., 2012). The MgO contents of the metabasalts range from 6.4$9.6 \mathrm{wt} \%$ and $\mathrm{Mg} \#\left[\left(\mathrm{MgO} /\left(\mathrm{MgO}+\mathrm{FeO}^{*}\right)\right]\right.$ varies from 52.7 to 64.8 , suggesting that some of these samples do not represent primitive melts. The metabasalts are similar to subalkaline mafic volcanic rocks (Fig. 4A), and have tholeiitic nature (Fig. 4B). 
Table 1. Composition and structural formula of pyroxene of Wadi Atalla El-Murr metabasalts, Central Eastern Desert, Egypt.

\begin{tabular}{|c|c|c|c|c|c|c|c|c|c|c|c|c|c|c|c|c|c|c|c|c|c|}
\hline Sample & E3 & E3 & E3 & E3 & E3 & E3 & E3 & E3 & E3 & E3 & E3 & E3 & E3 & E3 & E3 & E3 & E3 & E3 & E3 & E3 & E3 \\
\hline No. & 1 & 2 & 3 & 4 & 5 & 6 & 7 & 8 & 9 & 10 & 11 & 12 & 13 & 14 & 15 & 16 & 17 & 18 & 19 & 20 & 21 \\
\hline $\mathrm{SiO}_{2}$ & 52.47 & 52.46 & 53.03 & 53.08 & 52.98 & 53.40 & 52.93 & 53.61 & 53.00 & 54.10 & 53.00 & 53.29 & 53.33 & 52.92 & 53.40 & 52.20 & 52.28 & 52.06 & 52.37 & 52.26 & 53.45 \\
\hline $\mathrm{TiO}_{2}$ & 0.49 & 0.35 & 0.30 & 0.31 & 0.30 & 0.30 & 0.29 & 0.27 & 0.32 & 0.27 & 0.31 & 0.29 & 0.31 & 0.34 & 0.32 & 0.37 & 0.37 & 0.37 & 0.36 & 0.41 & 0.25 \\
\hline $\mathrm{Al}_{2} \mathrm{O}_{3}$ & 2.90 & 2.92 & 2.29 & 2.25 & 2.34 & 2.14 & 2.11 & 1.98 & 2.71 & 1.43 & 2.33 & 2.16 & 2.37 & 2.60 & 2.27 & 3.38 & 3.32 & 3.47 & 3.25 & 3.56 & 1.91 \\
\hline $\mathrm{FeO}$ & 8.14 & 6.45 & 5.99 & 5.94 & 6.11 & 6.02 & 5.90 & 6.50 & 6.24 & 8.96 & 6.41 & 6.39 & 6.68 & 6.32 & 6.54 & 6.42 & 6.58 & 6.53 & 6.49 & 6.62 & 6.26 \\
\hline $\mathrm{MnO}$ & 0.21 & 0.17 & 0.15 & 0.14 & 0.16 & 0.14 & 0.16 & 0.18 & 0.16 & 0.28 & 0.17 & 0.17 & 0.19 & 0.16 & 0.20 & 0.17 & 0.18 & 0.17 & 0.18 & 0.18 & 0.18 \\
\hline $\mathrm{MgO}$ & 18.48 & 17.47 & 17.47 & 17.63 & 17.58 & 17.62 & 17.69 & 18.42 & 17.51 & 20.57 & 17.94 & 18.01 & 18.29 & 17.76 & 18.29 & 17.18 & 17.06 & 17.47 & 17.12 & 17.28 & 18.06 \\
\hline $\mathrm{CaO}$ & 16.80 & 19.93 & 20.38 & 20.46 & 20.29 & 20.58 & 20.69 & 19.27 & 20.16 & 14.74 & 19.64 & 19.84 & 19.51 & 19.56 & 19.70 & 20.26 & 19.89 & 19.51 & 19.99 & 19.79 & 19.66 \\
\hline $\mathrm{K}_{2} \mathrm{O}$ & 0.00 & 0.00 & 0.00 & 0.00 & 0.00 & 0.00 & 0.01 & 0.00 & 0.00 & 0.01 & 0.01 & 0.00 & 0.00 & 0.00 & 0.00 & 0.01 & 0.00 & 0.00 & 0.01 & 0.00 & 0.01 \\
\hline $\mathrm{Na}_{2} \mathrm{O}$ & 0.19 & 0.23 & 0.15 & 0.20 & 0.19 & 0.17 & 0.21 & 0.19 & 0.20 & 0.12 & 0.19 & 0.21 & 0.17 & 0.24 & 0.16 & 0.24 & 0.24 & 0.24 & 0.26 & 0.23 & 0.19 \\
\hline $\mathrm{Cr}_{2} \mathrm{O}_{3}$ & 0.06 & 0.24 & 0.30 & 0.25 & 0.29 & 0.28 & 0.25 & 0.17 & 0.25 & 0.06 & 0.21 & 0.16 & 0.14 & 0.18 & 0.15 & 0.22 & 0.18 & 0.36 & 0.33 & 0.29 & 0.18 \\
\hline Total & 99.74 & 100.21 & 100.06 & 100.27 & 100.24 & 100.65 & 100.24 & 100.60 & 100.56 & 100.55 & 100.21 & 100.53 & 100.99 & 100.08 & 101.01 & 100.45 & 100.10 & 100.18 & 100.35 & 100.62 & 100.15 \\
\hline & & & & & & & & & & & & & & & & & & & & & \\
\hline \multicolumn{22}{|c|}{ Formula based on 6 oxygens } \\
\hline $\mathrm{Si}$ & 1.922 & 1.912 & 1.937 & 1.933 & 1.931 & 1.939 & 1.927 & 1.943 & 1.926 & 1.959 & 1.930 & 1.934 & 1.927 & 1.930 & 1.929 & 1.899 & 1.910 & 1.898 & 1.909 & 1.899 & 1.947 \\
\hline $\mathrm{Ti}$ & 0.014 & 0.010 & 0.008 & 0.008 & 0.008 & 0.008 & 0.008 & 0.007 & 0.009 & 0.007 & 0.008 & 0.008 & 0.008 & 0.009 & 0.009 & 0.010 & 0.010 & 0.010 & 0.010 & 0.011 & 0.007 \\
\hline $\mathrm{Al}(\mathrm{T})$ & 0.078 & 0.088 & 0.063 & 0.067 & 0.069 & 0.061 & 0.073 & 0.057 & 0.074 & 0.041 & 0.070 & 0.066 & 0.073 & 0.070 & 0.071 & 0.101 & 0.090 & 0.102 & 0.091 & 0.101 & 0.053 \\
\hline $\mathrm{Al}$ (M1) & 0.047 & 0.038 & 0.036 & 0.029 & 0.032 & 0.030 & 0.018 & 0.028 & 0.042 & 0.020 & 0.031 & 0.027 & 0.028 & 0.041 & 0.025 & 0.044 & 0.053 & 0.047 & 0.048 & 0.051 & 0.029 \\
\hline $\mathrm{Fe}^{3+}(\mathrm{T})$ & 0.000 & 0.000 & 0.000 & 0.000 & 0.000 & 0.000 & 0.000 & 0.000 & 0.000 & 0.000 & 0.000 & 0.000 & 0.000 & 0.000 & 0.000 & 0.000 & 0.000 & 0.000 & 0.000 & 0.000 & 0.000 \\
\hline $\mathrm{Fe}^{3+}(\mathrm{M} 1)$ & 0.015 & 0.040 & 0.013 & 0.028 & 0.026 & 0.019 & 0.047 & 0.023 & 0.022 & 0.013 & 0.029 & 0.033 & 0.037 & 0.022 & 0.035 & 0.047 & 0.028 & 0.041 & 0.032 & 0.035 & 0.018 \\
\hline $\mathrm{Fe}^{2+}$ & 0.234 & 0.156 & 0.170 & 0.153 & 0.161 & 0.164 & 0.132 & 0.175 & 0.168 & 0.258 & 0.166 & 0.161 & 0.165 & 0.171 & 0.162 & 0.148 & 0.173 & 0.158 & 0.166 & 0.166 & 0.173 \\
\hline $\mathrm{Mn}$ & 0.007 & 0.005 & 0.005 & 0.004 & 0.005 & 0.004 & 0.005 & 0.006 & 0.005 & 0.009 & 0.005 & 0.005 & 0.006 & 0.005 & 0.006 & 0.005 & 0.006 & 0.005 & 0.005 & 0.005 & 0.006 \\
\hline $\mathrm{K}$ & 0.000 & 0.000 & 0.000 & 0.000 & 0.000 & 0.000 & 0.000 & 0.000 & 0.000 & 0.000 & 0.001 & 0.000 & 0.000 & 0.000 & 0.000 & 0.001 & 0.000 & 0.000 & 0.000 & 0.000 & 0.000 \\
\hline $\mathrm{Na}$ & 0.013 & 0.016 & 0.011 & 0.014 & 0.013 & 0.012 & 0.015 & 0.013 & 0.014 & 0.009 & 0.013 & 0.015 & 0.012 & 0.017 & 0.011 & 0.017 & 0.017 & 0.017 & 0.018 & 0.016 & 0.014 \\
\hline $\mathrm{Cr}$ & 0.002 & 0.007 & 0.009 & 0.007 & 0.008 & 0.008 & 0.007 & 0.005 & 0.007 & 0.002 & 0.006 & 0.005 & 0.004 & 0.005 & 0.004 & 0.006 & 0.005 & 0.010 & 0.010 & 0.008 & 0.005 \\
\hline Total & 4.000 & 4.000 & 4.000 & 4.000 & 4.000 & 4.000 & 4.000 & 4.000 & 4.000 & 4.000 & 4.000 & 4.000 & 4.000 & 4.000 & 4.000 & 4.000 & 4.000 & 4.000 & 4.000 & 4.000 & 4.000 \\
\hline En & 52.44 & 49.20 & 49.12 & 49.32 & 49.27 & 49.12 & 49.19 & 51.14 & 49.20 & 56.59 & 50.18 & 50.10 & 50.57 & 50.09 & 50.49 & 48.48 & 48.54 & 49.56 & 48.60 & 48.93 & 50.44 \\
\hline Fs & 13.30 & 10.46 & 9.70 & 9.55 & 9.86 & 9.64 & 9.45 & 10.41 & 10.09 & 14.27 & 10.33 & 10.24 & 10.66 & 10.25 & 10.43 & 10.44 & 10.79 & 10.67 & 10.62 & 10.80 & 10.09 \\
\hline Wo & 34.26 & 40.34 & 41.18 & 41.13 & 40.87 & 41.24 & 41.35 & 38.45 & 40.71 & 29.14 & 39.49 & 39.66 & 38.77 & 39.65 & 39.08 & 41.09 & 40.67 & 39.78 & 40.78 & 40.27 & 39.47 \\
\hline
\end{tabular}


Table 1. Cont

\begin{tabular}{|c|c|c|c|c|c|c|c|c|c|c|c|c|c|c|c|c|c|c|c|c|c|}
\hline Sample & E3 & E3 & E3 & E3 & E3 & E3 & E3 & E3 & E3 & E24 & E24 & E24 & E24 & E24 & E24 & E24 & E24 & E24 & E24 & E24 & E24 \\
\hline No. & 22 & 23 & 24 & 25 & 26 & 27 & 28 & 29 & 30 & 31 & 32 & 33 & 34 & 35 & 36 & 37 & 38 & 39 & 40 & 41 & 42 \\
\hline $\mathrm{SiO}_{2}$ & 53.66 & 53.26 & 53.33 & 52.21 & 52.03 & 51.76 & 52.14 & 52.59 & 52.31 & 51.11 & 51.19 & 50.66 & 52.76 & 52.76 & 51.91 & 52.59 & 52.59 & 53.05 & 52.44 & 52.78 & 52.89 \\
\hline $\mathrm{TiO}_{2}$ & 0.27 & 0.30 & 0.30 & 0.39 & 0.38 & 0.38 & 0.39 & 0.29 & 0.39 & 0.70 & 0.75 & 0.70 & 0.39 & 0.37 & 0.54 & 0.43 & 0.38 & 0.39 & 0.48 & 0.34 & 0.36 \\
\hline $\mathrm{Al}_{2} \mathrm{O}_{3}$ & 1.76 & 2.20 & 2.11 & 3.39 & 3.46 & 3.60 & 3.52 & 2.85 & 3.28 & 2.45 & 2.26 & 2.13 & 1.86 & 1.89 & 2.36 & 1.84 & 1.98 & 2.03 & 2.25 & 2.18 & 2.19 \\
\hline $\mathrm{FeO}$ & 6.63 & 6.44 & 6.66 & 6.34 & 6.49 & 6.64 & 6.51 & 6.46 & 6.47 & 12.62 & 13.74 & 13.72 & 7.35 & 7.40 & 9.20 & 8.89 & 7.18 & 7.28 & 8.57 & 7.09 & 7.11 \\
\hline $\mathrm{MnO}$ & 0.19 & 0.17 & 0.17 & 0.17 & 0.16 & 0.15 & 0.15 & 0.20 & 0.19 & 0.36 & 0.35 & 0.36 & 0.17 & 0.18 & 0.25 & 0.24 & 0.17 & 0.18 & 0.21 & 0.21 & 0.23 \\
\hline $\mathrm{MgO}$ & 18.49 & 18.07 & 17.89 & 17.14 & 17.14 & 17.03 & 17.29 & 17.47 & 17.76 & 14.40 & 14.46 & 14.47 & 16.97 & 16.98 & 16.36 & 16.81 & 17.06 & 17.05 & 16.57 & 17.51 & 17.64 \\
\hline $\mathrm{CaO}$ & 19.20 & 19.97 & 19.46 & 19.60 & 20.22 & 19.63 & 19.47 & 19.62 & 19.00 & 18.40 & 17.83 & 17.61 & 19.89 & 19.79 & 18.88 & 19.57 & 19.94 & 20.21 & 19.34 & 19.44 & 19.42 \\
\hline $\mathrm{K}_{2} \mathrm{O}$ & 0.00 & 0.00 & 0.00 & 0.00 & 0.01 & 0.00 & 0.00 & 0.00 & 0.00 & 0.00 & 0.00 & 0.00 & 0.01 & 0.00 & 0.00 & 0.00 & 0.01 & 0.00 & 0.00 & 0.01 & 0.00 \\
\hline $\mathrm{Na}_{2} \mathrm{O}$ & 0.18 & 0.19 & 0.20 & 0.24 & 0.26 & 0.23 & 0.23 & 0.24 & 0.24 & 0.28 & 0.25 & 0.26 & 0.22 & 0.19 & 0.22 & 0.24 & 0.15 & 0.19 & 0.25 & 0.20 & 0.20 \\
\hline $\mathrm{Cr}_{2} \mathrm{O}_{3}$ & 0.13 & 0.21 & 0.12 & 0.25 & 0.22 & 0.24 & 0.27 & 0.33 & 0.48 & 0.02 & 0.03 & 0.00 & 0.09 & 0.09 & 0.03 & 0.03 & 0.12 & 0.09 & 0.04 & 0.12 & 0.13 \\
\hline Total & 100.5 & 100.81 & 100.25 & 99.74 & 100.38 & 99.67 & 99.96 & 100.05 & 100.12 & 100.33 & 100.87 & 99.91 & 99.71 & 99.65 & 99.74 & 100.64 & 99.58 & 100.46 & 100.15 & 99.87 & 100.17 \\
\hline & & & & & & & & & & & & & & & & & & & & & \\
\hline \multicolumn{22}{|c|}{ Formula based on 6 oxygens } \\
\hline $\mathrm{Si}$ & 1.947 & 1.928 & 1.943 & 1.913 & 1.894 & 1.899 & 1.906 & 1.921 & 1.907 & 1.908 & 1.907 & 1.904 & 1.942 & 1.944 & 1.921 & 1.926 & 1.938 & 1.938 & 1.929 & 1.935 & 1.933 \\
\hline $\mathrm{Ti}$ & 0.007 & 0.008 & 0.008 & 0.011 & 0.010 & 0.011 & 0.011 & 0.008 & 0.011 & 0.020 & 0.021 & 0.020 & 0.011 & 0.010 & 0.015 & 0.012 & 0.011 & 0.011 & 0.013 & 0.009 & 0.010 \\
\hline $\mathrm{Al}(\mathrm{T})$ & 0.053 & 0.072 & 0.057 & 0.087 & 0.106 & 0.101 & 0.094 & 0.079 & 0.093 & 0.092 & 0.093 & 0.094 & 0.058 & 0.056 & 0.079 & 0.074 & 0.062 & 0.062 & 0.071 & 0.065 & 0.067 \\
\hline $\mathrm{Al}(\mathrm{M} 1)$ & 0.023 & 0.022 & 0.034 & 0.059 & 0.043 & 0.055 & 0.057 & 0.043 & 0.048 & 0.016 & 0.006 & 0.000 & 0.023 & 0.026 & 0.024 & 0.005 & 0.024 & 0.026 & 0.027 & 0.029 & 0.027 \\
\hline $\mathrm{Fe}^{3+}(\mathrm{T})$ & 0.000 & 0.000 & 0.000 & 0.000 & 0.000 & 0.000 & 0.000 & 0.000 & 0.000 & 0.000 & 0.000 & 0.002 & 0.000 & 0.000 & 0.000 & 0.000 & 0.000 & 0.000 & 0.000 & 0.000 & 0.000 \\
\hline $\begin{array}{l}\mathrm{Fe}^{3+} \\
\text { (M1) }\end{array}$ & 0.025 & 0.041 & 0.017 & 0.016 & 0.055 & 0.035 & 0.025 & 0.028 & 0.027 & 0.056 & 0.062 & 0.075 & 0.026 & 0.020 & 0.040 & 0.062 & 0.024 & 0.025 & 0.035 & 0.029 & 0.031 \\
\hline $\mathrm{Fe}^{2+}$ & 0.177 & 0.154 & 0.186 & 0.178 & 0.143 & 0.169 & 0.174 & 0.170 & 0.170 & 0.338 & 0.366 & 0.354 & 0.200 & 0.208 & 0.245 & 0.210 & 0.197 & 0.197 & 0.229 & 0.188 & 0.186 \\
\hline $\mathrm{Ca}$ & 0.747 & 0.775 & 0.760 & 0.769 & 0.789 & 0.772 & 0.762 & 0.768 & 0.742 & 0.736 & 0.712 & 0.709 & 0.785 & 0.781 & 0.749 & 0.768 & 0.787 & 0.791 & 0.762 & 0.763 & 0.760 \\
\hline $\mathrm{K}$ & 0.000 & 0.000 & 0.000 & 0.000 & 0.001 & 0.000 & 0.000 & 0.000 & 0.000 & 0.000 & 0.000 & 0.000 & 0.001 & 0.000 & 0.000 & 0.000 & 0.001 & 0.000 & 0.000 & 0.000 & 0.000 \\
\hline $\mathrm{Na}$ & 0.013 & 0.014 & 0.014 & 0.017 & 0.019 & 0.016 & 0.016 & 0.017 & 0.017 & 0.021 & 0.018 & 0.019 & 0.016 & 0.014 & 0.016 & 0.017 & 0.011 & 0.013 & 0.018 & 0.014 & 0.014 \\
\hline $\mathrm{Cr}$ & 0.004 & 0.006 & 0.004 & 0.007 & 0.006 & 0.007 & 0.008 & 0.009 & 0.014 & 0.001 & 0.001 & 0.000 & 0.003 & 0.003 & 0.001 & 0.001 & 0.003 & 0.003 & 0.001 & 0.003 & 0.004 \\
\hline Total & 4.000 & 4.000 & 4.000 & 4.000 & 4.000 & 4.000 & 4.000 & 4.000 & 4.000 & 4.000 & 4.000 & 4.000 & 4.000 & 4.000 & 4.000 & 4.000 & 4.000 & 4.000 & 4.000 & 4.000 & 4.000 \\
\hline En & 51.20 & 50.01 & 50.10 & 49.14 & 48.41 & 48.73 & 49.37 & 49.48 & 50.52 & 41.25 & 41.10 & 41.31 & 47.82 & 47.89 & 46.44 & 46.69 & 48.03 & 47.67 & 46.81 & 49.21 & 49.39 \\
\hline Fs & 10.60 & 10.27 & 10.74 & 10.47 & 10.54 & 10.90 & 10.67 & 10.58 & 10.63 & 20.86 & 22.48 & 22.55 & 11.90 & 11.99 & 15.05 & 14.24 & 11.62 & 11.71 & 13.92 & 11.52 & 11.53 \\
\hline Wo & 38.21 & 39.72 & 39.16 & 40.39 & 41.05 & 40.37 & 39.96 & 39.94 & 38.84 & 37.89 & 36.42 & 36.13 & 40.28 & 40.12 & 38.52 & 39.07 & 40.35 & 40.61 & 39.27 & 39.27 & 39.08 \\
\hline
\end{tabular}


Maurice, A. E.

Table 1. Cont.

\begin{tabular}{|c|c|c|c|c|c|c|c|c|c|c|c|c|c|c|c|c|c|c|c|c|c|}
\hline Sample & E24 & E24 & E24 & E24 & E24 & E24 & E24 & E24 & E24 & E24 & E24 & E24 & E24 & E24 & E24 & E24 & E24 & E24 & E24 & E24 & E24 \\
\hline No. & 43 & 44 & 45 & 46 & 47 & 48 & 49 & 50 & 51 & 52 & 53 & 54 & 55 & 56 & 57 & 58 & 59 & 60 & 61 & 62 & 63 \\
\hline $\mathrm{SiO}_{2}$ & 52.80 & 51.98 & 52.36 & 52.49 & 51.93 & 52.25 & 52.96 & 52.68 & 52.78 & 52.89 & 52.83 & 52.84 & 52.89 & 52.63 & 52.31 & 52.32 & 51.97 & 52.86 & 52.96 & 53.10 & 52.78 \\
\hline $\mathrm{TiO}_{2}$ & 0.34 & 0.38 & 0.40 & 0.38 & 0.39 & 0.34 & 0.36 & 0.38 & 0.34 & 0.33 & 0.35 & 0.37 & 0.34 & 0.41 & 0.42 & 0.40 & 0.36 & 0.34 & 0.34 & 0.36 & 0.35 \\
\hline $\mathrm{Al}_{2} \mathrm{O}_{3}$ & 2.20 & 2.59 & 2.59 & 2.47 & 2.54 & 2.54 & 2.16 & 2.32 & 2.22 & 2.09 & 2.14 & 2.13 & 2.13 & 2.77 & 2.81 & 2.25 & 2.29 & 2.32 & 2.17 & 2.24 & 1.98 \\
\hline $\mathrm{FeO}$ & 7.02 & 7.21 & 7.22 & 6.94 & 7.13 & 7.78 & 7.07 & 7.14 & 7.10 & 7.22 & 7.17 & 7.24 & 7.11 & 7.07 & 7.05 & 7.21 & 7.45 & 6.74 & 6.76 & 7.04 & 7.06 \\
\hline $\mathrm{MnO}$ & 0.17 & 0.18 & 0.19 & 0.21 & 0.19 & 0.21 & 0.19 & 0.18 & 0.18 & 0.23 & 0.19 & 0.19 & 0.23 & 0.18 & 0.18 & 0.20 & 0.18 & 0.18 & 0.16 & 0.18 & 0.24 \\
\hline $\mathrm{MgO}$ & 17.38 & 17.41 & 17.29 & 17.30 & 17.26 & 17.15 & 17.29 & 17.23 & 17.35 & 17.59 & 17.38 & 17.59 & 17.61 & 17.12 & 17.12 & 17.38 & 16.83 & 17.53 & 17.53 & 17.31 & 17.26 \\
\hline $\mathrm{CaO}$ & 19.76 & 19.75 & 19.80 & 20.05 & 20.15 & 18.74 & 19.43 & 19.49 & 19.69 & 19.68 & 19.71 & 19.96 & 19.88 & 19.95 & 19.77 & 20.04 & 20.27 & 20.10 & 19.76 & 19.84 & 20.14 \\
\hline $\mathrm{K}_{2} \mathrm{O}$ & 0.00 & 0.00 & 0.01 & 0.00 & 0.00 & 0.00 & 0.00 & 0.01 & 0.01 & 0.00 & 0.00 & 0.00 & 0.00 & 0.00 & 0.01 & 0.00 & 0.00 & 0.00 & 0.00 & 0.00 & 0.01 \\
\hline $\mathrm{Na}_{2} \mathrm{O}$ & 0.21 & 0.20 & 0.20 & 0.19 & 0.22 & 0.21 & 0.20 & 0.20 & 0.23 & 0.26 & 0.18 & 0.22 & 0.21 & 0.25 & 0.22 & 0.23 & 0.26 & 0.23 & 0.21 & 0.18 & 0.19 \\
\hline $\mathrm{Cr}_{2} \mathrm{O}_{3}$ & 0.12 & 0.17 & 0.21 & 0.17 & 0.18 & 0.14 & 0.14 & 0.14 & 0.12 & 0.12 & 0.11 & 0.11 & 0.12 & 0.14 & 0.13 & 0.18 & 0.18 & 0.22 & 0.16 & 0.16 & 0.14 \\
\hline Total & 100.00 & 99.88 & 100.28 & 100.20 & 99.99 & 99.36 & 99.80 & 99.76 & 100.01 & 100.41 & 100.07 & 100.64 & 100.52 & 100.52 & 100.02 & 100.21 & 99.80 & 100.52 & 100.05 & 100.41 & 100.15 \\
\hline \multirow{2}{*}{\multicolumn{22}{|c|}{ Formula based on 6 oxygens }} \\
\hline & & & & & & & & & & & & & & & & & & & & & \\
\hline $\mathrm{Si}$ & 1.933 & 1.905 & 1.913 & 1.918 & 1.902 & 1.929 & 1.944 & 1.935 & 1.933 & 1.928 & 1.934 & 1.922 & 1.926 & 1.918 & 1.916 & 1.912 & 1.911 & 1.924 & 1.937 & 1.938 & 1.932 \\
\hline $\mathrm{Ti}$ & 0.009 & 0.011 & 0.011 & 0.010 & 0.011 & 0.010 & 0.010 & 0.010 & 0.009 & 0.009 & 0.010 & 0.010 & 0.009 & 0.011 & 0.011 & 0.011 & 0.010 & 0.009 & 0.009 & 0.010 & 0.010 \\
\hline $\mathrm{Al}(\mathrm{T})$ & 0.067 & 0.095 & 0.087 & 0.082 & 0.098 & 0.071 & 0.056 & 0.065 & 0.067 & 0.072 & 0.066 & 0.078 & 0.074 & 0.082 & 0.084 & 0.088 & 0.089 & 0.076 & 0.063 & 0.062 & 0.068 \\
\hline $\mathrm{Al}(\mathrm{M} 1)$ & 0.028 & 0.017 & 0.025 & 0.025 & 0.011 & 0.040 & 0.038 & 0.035 & 0.028 & 0.018 & 0.027 & 0.014 & 0.017 & 0.037 & 0.037 & 0.009 & 0.010 & 0.023 & 0.030 & 0.035 & 0.017 \\
\hline $\mathrm{Fe}^{3+}(\mathrm{T})$ & 0.000 & 0.000 & 0.000 & 0.000 & 0.000 & 0.000 & 0.000 & 0.000 & 0.000 & 0.000 & 0.000 & 0.000 & 0.000 & 0.000 & 0.000 & 0.000 & 0.000 & 0.000 & 0.000 & 0.000 & 0.000 \\
\hline $\begin{array}{l}\mathrm{Fe}^{3+} \\
\text { (M1) }\end{array}$ & 0.031 & 0.066 & 0.048 & 0.045 & 0.076 & 0.023 & 0.008 & 0.019 & 0.033 & 0.051 & 0.029 & 0.056 & 0.049 & 0.036 & 0.037 & 0.068 & 0.072 & 0.045 & 0.025 & 0.015 & 0.042 \\
\hline $\mathrm{Fe}^{2+}$ & 0.184 & 0.155 & 0.172 & 0.167 & 0.143 & 0.217 & 0.209 & 0.200 & 0.184 & 0.169 & 0.190 & 0.164 & 0.167 & 0.179 & 0.179 & 0.152 & 0.157 & 0.160 & 0.182 & 0.200 & 0.174 \\
\hline $\mathrm{Ca}$ & 0.775 & 0.776 & 0.775 & 0.785 & 0.791 & 0.741 & 0.764 & 0.767 & 0.772 & 0.769 & 0.773 & 0.778 & 0.776 & 0.779 & 0.776 & 0.785 & 0.799 & 0.784 & 0.774 & 0.776 & 0.790 \\
\hline $\mathrm{K}$ & 0.000 & 0.000 & 0.000 & 0.000 & 0.000 & 0.000 & 0.000 & 0.000 & 0.000 & 0.000 & 0.000 & 0.000 & 0.000 & 0.000 & 0.000 & 0.000 & 0.000 & 0.000 & 0.000 & 0.000 & 0.001 \\
\hline $\mathrm{Na}$ & 0.015 & 0.014 & 0.014 & 0.014 & 0.015 & 0.015 & 0.014 & 0.014 & 0.016 & 0.018 & 0.013 & 0.015 & 0.015 & 0.018 & 0.016 & 0.017 & 0.019 & 0.016 & 0.015 & 0.012 & 0.014 \\
\hline $\mathrm{Cr}$ & 0.003 & 0.005 & 0.006 & 0.005 & 0.005 & 0.004 & 0.004 & 0.004 & 0.004 & 0.003 & 0.003 & 0.003 & 0.003 & 0.004 & 0.004 & 0.005 & 0.005 & 0.006 & 0.005 & 0.005 & 0.004 \\
\hline Total & 4.000 & 4.000 & 4.000 & 4.000 & 4.000 & 4.000 & 4.000 & 4.000 & 4.000 & 4.000 & 4.000 & 4.000 & 4.000 & 4.000 & 4.000 & 4.000 & 4.000 & 4.000 & 4.000 & 4.000 & 4.000 \\
\hline En & 48.79 & 48.69 & 48.46 & 48.43 & 48.14 & 48.86 & 48.94 & 48.75 & 48.76 & \begin{tabular}{|l|l|}
48.98 \\
\end{tabular} & 48.71 & 48.72 & 48.89 & 48.19 & 48.38 & 48.35 & 47.17 & 48.88 & 49.22 & 48.59 & 48.17 \\
\hline Fs & 11.33 & 11.60 & 11.66 & 11.23 & 11.46 & 12.77 & 11.54 & 11.62 & 11.48 & 11.64 & 11.58 & 11.55 & 11.44 & 11.45 & 11.47 & 11.57 & 12.00 & 10.83 & 10.90 & 11.38 & 11.44 \\
\hline Wo & 39.87 & 39.70 & 39.88 & 40.34 & 40.39 & 38.37 & 39.53 & 39.63 & 39.77 & 39.38 & 39.70 & 39.73 & 39.67 & 40.36 & 40.15 & 40.07 & 40.83 & 40.28 & 39.88 & 40.03 & 40.39 \\
\hline
\end{tabular}


Table 1. Cont.

\begin{tabular}{|c|c|c|c|c|c|c|c|c|c|c|c|c|c|c|c|c|c|c|c|c|c|c|}
\hline Sample & E24 & E24 & E24 & E24 & E24 & E24 & E24 & E24 & E24 & E24 & E24 & E24 & E24 & E24 & E24 & E24 & E24 & E24 & E24 & E24 & E24 & E24 \\
\hline No. & 64 & 65 & 66 & 67 & 68 & 69 & 70 & 71 & 72 & 73 & 74 & 75 & 76 & 77 & 78 & 79 & 80 & 81 & 82 & 83 & 84 & 85 \\
\hline $\mathrm{SiO}_{2}$ & 52.78 & 53.00 & 52.89 & 52.96 & 52.93 & 52.82 & 52.90 & 52.82 & 52.74 & 52.82 & 51.38 & 52.10 & 52.34 & 52.14 & 52.81 & 52.48 & 52.69 & 52.57 & 52.57 & 52.60 & 51.29 & 51.18 \\
\hline $\mathrm{TiO}_{2}$ & 0.36 & 0.38 & 0.40 & 0.41 & 0.38 & 0.40 & 0.43 & 0.45 & 0.44 & 0.45 & 0.55 & 0.52 & 0.50 & 0.46 & 0.48 & 0.46 & 0.48 & 0.45 & 0.45 & 0.45 & 0.70 & 0.72 \\
\hline $\mathrm{Al}_{2} \mathrm{O}_{3}$ & 1.98 & 2.15 & 1.87 & 2.05 & 1.95 & 2.03 & 2.03 & 2.02 & 2.00 & 2.00 & 2.04 & 1.77 & 1.64 & 1.87 & 1.64 & 1.61 & 1.64 & 1.68 & 1.85 & 1.86 & 2.11 & 2.07 \\
\hline $\mathrm{FeO}$ & 7.14 & 7.13 & 7.33 & 7.13 & 7.35 & 7.80 & 7.97 & 8.32 & 8.47 & 8.83 & 13.48 & 13.92 & 12.70 & 12.40 & 111.68 & 11.75 & 11.60 & 11.57 & 11.99 & 12.20 & 13.71 & 13.66 \\
\hline $\mathrm{MnO}$ & 0.15 & 0.21 & 0.20 & 0.17 & 0.19 & 0.19 & 0.20 & 0.22 & 0.21 & 0.23 & 0.38 & 0.36 & 0.35 & 0.34 & 0.30 & 0.32 & 0.31 & 0.32 & 0.34 & 0.34 & 0.35 & 0.39 \\
\hline $\mathrm{MgO}$ & 17.21 & 16.96 & 16.94 & 16.91 & 17.08 & 16.88 & 16.77 & 16.78 & 16.71 & 16.74 & 15.38 & 16.17 & 16.45 & 16.60 & 16.86 & 16.81 & 16.91 & 16.86 & 16.84 & 16.79 & 14.34 & 14.27 \\
\hline $\mathrm{CaO}$ & 20.04 & 20.06 & 20.14 & 20.53 & 20.56 & 20.36 & 20.14 & 20.07 & 19.72 & 19.29 & 16.58 & 15.85 & 16.25 & 16.73 & 16.91 & 17.07 & $\begin{array}{l}17.31 \\
\end{array}$ & 17.15 & 16.43 & 16.28 & 17.89 & 17.69 \\
\hline $\mathrm{K}_{2} \mathrm{O}$ & 0.00 & 0.00 & 0.00 & 0.00 & 0.00 & 0.00 & $\begin{array}{l}0.01 \\
\end{array}$ & $\begin{array}{l}0.01 \\
\end{array}$ & 0.00 & 0.01 & 0.01 & 0.00 & 0.00 & 0.00 & 0.00 & 0.00 & 0.01 & 0.01 & 0.00 & 0.00 & 0.00 & 0.01 \\
\hline $\mathrm{Na}_{2} \mathrm{O}$ & 0.18 & 0.19 & 0.19 & 0.18 & 0.18 & 0.21 & 0.19 & 0.24 & 0.21 & 0.21 & 0.25 & 0.20 & 0.23 & 0.19 & 0.21 & 0.19 & 0.25 & 0.20 & 0.22 & 0.22 & 0.27 & 0.25 \\
\hline $\mathrm{Cr}_{2} \mathrm{O}_{3}$ & 0.14 & 0.16 & 0.04 & 0.10 & 0.11 & 0.06 & 0.05 & 0.03 & 0.02 & 0.00 & 0.00 & 0.00 & 0.02 & 0.00 & 0.00 & 0.00 & 0.00 & 0.00 & 0.02 & 0.02 & 0.02 & 0.00 \\
\hline Total & 99.99 & 100.25 & 99.99 & 100.43 & 100.74 & 100.76 & 100.69 & 100.95 & 100.53 & 100.58 & 100.04 & 100.89 & 100.48 & 100.73 & 100.88 & 100.69 & 101.19 & 100.81 & 100.70 & 100.75 & 100.69 & 100.23 \\
\hline & & & & & & & & & & & & & & & & & & & & & & \\
\hline \multicolumn{23}{|c|}{ Formula based on 6 oxygens } \\
\hline $\mathrm{Si}$ & 1.935 & 1.941 & 1.942 & 1.936 & 1.929 & 1.927 & 1.933 & 1.926 & 1.933 & 1.936 & 1.921 & 1.930 & 1.939 & 1.924 & 1.942 & 1.934 & 1.930 & 1.934 & 1.937 & 1.939 & 1.914 & 1.920 \\
\hline $\mathrm{Ti}$ & 0.010 & 0.011 & 0.011 & 0.011 & 0.010 & 0.011 & 0.012 & 0.012 & 0.012 & 0.013 & 0.015 & 0.015 & 0.014 & 0.013 & 0.013 & 0.013 & 0.013 & 0.012 & 0.012 & 0.012 & 0.020 & 0.020 \\
\hline $\mathrm{Al}(\mathrm{T})$ & 0.065 & 0.059 & 0.058 & 0.064 & 0.071 & 0.073 & 0.067 & 0.074 & 0.067 & 0.064 & 0.079 & 0.070 & 0.061 & 0.076 & 0.058 & 0.066 & 0.070 & 0.066 & 0.063 & 0.061 & 0.086 & 0.080 \\
\hline $\begin{array}{l}\text { Al } \\
\text { (M1) }\end{array}$ & 0.021 & 0.033 & 0.023 & 0.024 & 0.013 & 0.014 & 0.021 & 013 & .019 & 0.022 & 0.011 & 0.007 & 0.011 & 0.006 & 0.013 & 0.004 & 0.001 & 0.007 & 0.018 & 0.019 & 0.007 & 0.011 \\
\hline $\begin{array}{l}\mathrm{Fe}^{3+} \\
(\mathrm{T}) \\
\end{array}$ & 0.000 & 0.000 & 0.000 & 0.000 & 0.000 & 0.000 & 0.000 & 0.000 & 0.000 & 0.000 & 0.000 & 0.000 & 0.000 & 0.000 & 0.000 & 0.000 & 0.000 & 0.000 & 0.000 & 0.000 & 0.000 & 0.000 \\
\hline $\mathrm{Fe}^{3+}$ & 0.033 & 0.014 & & 027 & 048 & .049 & .034 & 0.053 & 038 & .031 & 0.054 & 0.048 & 0.038 & 0.058 & 0.033 & 0.050 & 0.060 & 0.048 & 0.035 & 0.032 & 0.058 & 0.046 \\
\hline $\mathrm{Mn}$ & 0.005 & 0.007 & 0.006 & 0.005 & 0.006 & 0.006 & 0.006 & 0.007 & 0.006 & 0.007 & 0.012 & 0.011 & 0.011 & 0.011 & 0.009 & 0.010 & 0.010 & 0.010 & 0.011 & 0.011 & 0.011 & 0.012 \\
\hline $\mathrm{Mg}$ & 0.941 & 0.926 & 0.927 & 0.922 & 0.928 & 0.918 & 0.914 & 0.912 & 0.913 & 0.915 & 0.857 & 0.893 & 0.909 & 0.913 & 0.924 & 0.924 & 0.924 & 0.925 & 0.925 & 0.923 & 0.798 & 0.798 \\
\hline $\mathrm{Ca}$ & 0.787 & 0.787 & 0.792 & 0.804 & 0.803 & 0.796 & $\begin{array}{l}0.789 \\
\end{array}$ & 0.784 & 0.774 & 0.758 & 0.664 & 0.629 & 0.645 & 0.662 & 0.666 & 0.674 & 0.679 & 0.676 & 0.649 & 0.643 & 0.715 & 0.711 \\
\hline $\mathrm{K}$ & 0.000 & 0.000 & 0.000 & 0.000 & 0.000 & 0.000 & 0.001 & 0.000 & 0.000 & 0.000 & 0.000 & 0.000 & 0.000 & 0.000 & 0.000 & 0.000 & 0.000 & 0.000 & 0.000 & 0.000 & 0.000 & 0.000 \\
\hline $\mathrm{Na}$ & 0.013 & 0.014 & 0.013 & 0.013 & 0.013 & 0.015 & 0.013 & 0.017 & 0.015 & 0.015 & 0.018 & 0.014 & 0.017 & 0.013 & 0.015 & 0.014 & 0.017 & 0.014 & 0.015 & 0.015 & 0.020 & 0.018 \\
\hline $\mathrm{Cr}$ & 0.004 & 0.005 & 0.001 & 0.003 & 0.003 & 0.002 & 0.001 & 0.001 & 0.001 & 0.000 & 0.000 & 0.000 & 0.000 & 0.000 & 0.000 & 0.000 & 0.000 & 0.000 & 0.001 & 0.000 & 0.001 & 0.000 \\
\hline Total & 4.000 & 4.000 & 4.000 & 4.000 & 4.000 & 4.000 & 4.000 & 4.000 & 4.000 & 4.000 & 4.000 & 4.000 & 4.000 & 4.000 & 4.000 & 4.000 & 4.000 & 4.000 & 4.000 & 4.000 & 4.000 & 4.000 \\
\hline En & 48.20 & $\begin{array}{l}47.78 \\
\end{array}$ & 47.53 & 47.29 & $\begin{array}{l}47.33 \\
\end{array}$ & $\begin{array}{l}46.89 \\
\end{array}$ & 46.80 & $\begin{array}{l}46.61 \\
\end{array}$ & 46.74 & 46.91 & 43.85 & 45.46 & 46.40 & 46.40 & $\begin{array}{l}47.18 \\
\end{array}$ & 46.88 & 46.93 & 47.02 & 47.35 & 47.26 & 40.87 & 40.93 \\
\hline Fs & 11.46 & 11.61 & 11.85 & 11.46 & 11.72 & 12.46 & 12.80 & $\begin{array}{l}13.32 \\
\end{array}$ & $\begin{array}{l}13.62 \\
\end{array}$ & 14.25 & 22.17 & 22.52 & 20.65 & 19.98 & 18.81 & 18.90 & 18.55 & 18.61 & 19.45 & 19.81 & 22.49 & 22.61 \\
\hline Wo & 40.34 & 40.61 & 40.61 & 41.26 & 40.95 & 40.65 & 40.40 & 40.07 & 39.64 & 38.85 & 33.98 & 32.02 & 32.94 & 33.61 & 34.01 & 34.22 & 34.52 & 34.37 & 33.20 & 32.93 & 36.64 & 36.46 \\
\hline
\end{tabular}


Maurice, A. E.

Table 2. Composition and structural formula of plagioclase of Wadi Atalla El-Murr metabasalts, Central Eastern Desert, Egypt.

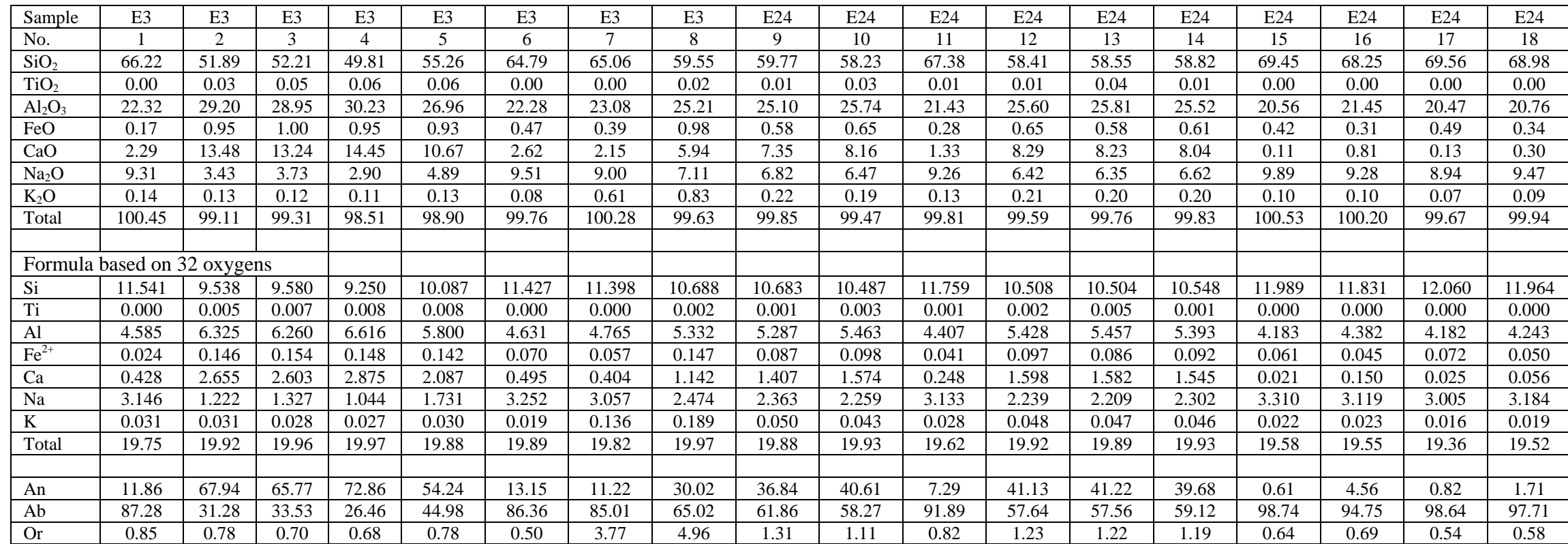


Petrology and geochemistry of Wadi Atalla El-Murr

Table 2. Cont.

\begin{tabular}{|c|c|c|c|c|c|c|c|c|c|c|c|c|c|c|c|c|c|c|}
\hline $\begin{array}{l}\text { No. } \\
\mathrm{SiO}_{2}\end{array}$ & $\begin{array}{c}19 \\
54.79\end{array}$ & $\begin{array}{c}20 \\
56.30\end{array}$ & $\begin{array}{c}21 \\
69.49\end{array}$ & $\begin{array}{c}22 \\
69.46\end{array}$ & $\begin{array}{c}23 \\
69.74\end{array}$ & $\begin{array}{c}24 \\
66.92\end{array}$ & $\frac{25}{57.82}$ & $\begin{array}{c}26 \\
57.11\end{array}$ & $\frac{27}{57.32}$ & $\begin{array}{c}28 \\
57.31\end{array}$ & $\begin{array}{c}29 \\
57.29\end{array}$ & $\begin{array}{c}30 \\
64.49\end{array}$ & $\begin{array}{c}31 \\
67.33\end{array}$ & $\begin{array}{c}32 \\
68.39\end{array}$ & $\frac{33}{58.05}$ & $\begin{array}{c}34 \\
66.49\end{array}$ & $\frac{35}{56.95}$ & $\begin{array}{c}36 \\
67.09\end{array}$ \\
\hline $\mathrm{TiO}_{2}$ & 0.05 & 0.01 & 0.00 & 0.00 & 0.01 & 0.01 & 0.00 & 0.01 & 0.02 & 0.01 & 0.02 & 0.00 & 0.00 & 0.00 & 0.02 & 0.01 & 0.01 & 0.01 \\
\hline $\mathrm{Al}_{2} \mathrm{O}_{3}$ & 27.67 & 26.99 & 20.61 & 20.80 & 20.45 & 21.94 & 26.22 & 26.59 & 26.59 & 26.43 & 26.40 & 23.13 & 20.81 & 20.89 & 25.96 & 21.59 & 26.67 & 21.25 \\
\hline $\mathrm{FeO}$ & 0.67 & 0.53 & 0.28 & 0.32 & 0.32 & 0.28 & 0.51 & 0.49 & 0.45 & 0.38 & 0.48 & 0.36 & 0.15 & 0.21 & 0.68 & 0.41 & 0.65 & 0.34 \\
\hline $\mathrm{CaO}$ & 10.93 & 9.57 & 0.12 & 0.11 & 0.34 & 1.97 & 8.42 & 8.92 & 8.79 & 7.83 & 8.67 & 2.80 & 0.51 & 0.24 & 8.77 & 2.15 & 9.37 & 1.82 \\
\hline $\mathrm{Na}_{2} \mathrm{O}$ & 5.00 & 5.63 & 9.49 & 9.67 & 8.85 & 6.83 & 6.29 & 6.04 & 6.03 & 6.00 & 6.10 & 8.31 & 9.63 & 9.02 & 6.06 & 8.87 & 5.79 & 8.88 \\
\hline $\mathrm{K}_{2} \mathrm{O}$ & 0.13 & 0.11 & 0.10 & 0.07 & 0.08 & 0.15 & 0.18 & 0.17 & 0.17 & 0.16 & 0.18 & 0.39 & 0.06 & 0.05 & 0.18 & 0.29 & 0.18 & 0.26 \\
\hline Total & 99.24 & 99.14 & 100.10 & 100.43 & 99.78 & 98.10 & 99.44 & 99.33 & 99.37 & 98.12 & 99.14 & 99.48 & 98.50 & 98.80 & 99.73 & 99.81 & 99.62 & 99.65 \\
\hline \multicolumn{19}{|c|}{ Formula based on 32 oxygens } \\
\hline $\mathrm{Si}$ & 9.975 & 10.205 & 12.017 & 11.983 & 12.070 & 11.786 & 10.415 & 10.315 & 10.338 & 10.421 & 10.358 & 11.372 & 11.871 & 11.964 & 10.437 & 11.653 & 10.274 & 11.747 \\
\hline $\mathrm{Ti}$ & 0.007 & 0.001 & 0.000 & 0.000 & 0.001 & 0.001 & 0.000 & 0.001 & 0.003 & 0.001 & 0.003 & 0.000 & 0.000 & 0.000 & 0.003 & 0.001 & 0.002 & 0.001 \\
\hline $\mathrm{Al}$ & 5.937 & 5.765 & 4.200 & 4.229 & 4.171 & 4.554 & 5.566 & 5.660 & 5.652 & 5.664 & 5.625 & 4.807 & 4.324 & 4.307 & 5.501 & 4.459 & 5.670 & 4.385 \\
\hline $\mathrm{Fe}^{2+}$ & 0.102 & 0.080 & 0.041 & 0.046 & 0.046 & 0.041 & 0.076 & 0.074 & 0.067 & 0.058 & 0.073 & 0.053 & 0.022 & 0.030 & 0.103 & 0.060 & 0.099 & 0.050 \\
\hline $\mathrm{Ca}$ & 2.132 & 1.858 & 0.023 & 0.021 & 0.063 & 0.372 & 1.625 & 1.726 & 1.698 & 1.525 & 1.679 & 0.529 & 0.097 & 0.045 & 1.689 & 0.404 & 1.811 & 0.341 \\
\hline $\mathrm{Na}$ & 1.765 & 1.978 & 3.182 & 3.234 & 2.969 & 2.332 & 2.197 & 2.115 & 2.108 & 2.115 & 2.138 & 2.841 & 3.292 & 3.059 & 2.112 & 3.014 & 2.025 & 3.014 \\
\hline K & 0.030 & 0.026 & 0.023 & 0.016 & 0.018 & 0.034 & 0.042 & 0.039 & 0.040 & 0.036 & 0.042 & 0.089 & 0.014 & 0.011 & 0.042 & 0.066 & 0.041 & 0.059 \\
\hline Total & 19.95 & 19.91 & 19.49 & 19.53 & 19.34 & 19.12 & 19.92 & 19.93 & 19.91 & 19.82 & 19.92 & 19.69 & 19.62 & 19.42 & 19.89 & 19.66 & 19.92 & 19.60 \\
\hline An & 54.30 & 48.12 & 0.70 & 0.63 & 2.06 & 13.58 & 42.05 & 44.49 & 44.16 & 41.49 & 43.51 & 15.29 & 2.84 & 1.45 & 43.95 & 11.59 & 46.71 & 10.00 \\
\hline $\mathrm{Ab}$ & 44.95 & 51.22 & 98.60 & 98.89 & 97.35 & 85.18 & 56.85 & 54.51 & 54.82 & 57.53 & 55.40 & 82.14 & 96.75 & 98.21 & 54.95 & 86.53 & 52.23 & 88.28 \\
\hline Or & 0.76 & 0.66 & 0.71 & 0.48 & 0.60 & 1.24 & 1.09 & 1.00 & 1.03 & 0.99 & 1.08 & 2.56 & 0.40 & 0.34 & 1.10 & 1.88 & 1.06 & 1.72 \\
\hline
\end{tabular}


Maurice, A. E.

Table 3. Composition of Wadi Atalla El-Murr metabasalts, Central Eastern Desert

\begin{tabular}{|c|c|c|c|c|c|c|c|c|c|c|c|c|c|}
\hline Sample & E3A & E3B & E8C & E7B2 & E15A & E15B & E18A & E18B & E24A & E24B & E25B & E28B1 & E28B2 \\
\hline $\mathrm{SiO}_{2}$ & 49.38 & 49.12 & 50.33 & 51.91 & 47.08 & 47.27 & 49.72 & 44.60 & 48.53 & 48.64 & 48.15 & 48.11 & 47.71 \\
\hline $\mathrm{TiO}_{2}$ & $\begin{array}{l}1.15 \\
\end{array}$ & 1.15 & 1.11 & $\begin{array}{ll}1.08 \\
\end{array}$ & 1.06 & $\begin{array}{l}1.11 \\
\end{array}$ & $\begin{array}{ll}1.06 \\
\end{array}$ & $\begin{array}{l}1.19 \\
\end{array}$ & 1.25 & 1.27 & 0.88 & $\begin{array}{l}0.88 \\
\end{array}$ & 0.89 \\
\hline $\mathrm{Al}_{2} \mathrm{O}_{3}$ & 13.57 & 13.83 & 13.76 & 14.16 & 15.32 & 14.51 & 13.44 & 13.15 & 14.33 & 14.49 & 15.03 & 14.75 & 14.42 \\
\hline $\mathrm{Fe}_{2} \mathrm{O}_{3}$ & 12.21 & 12.14 & 11.09 & 10.59 & 12.08 & 11.97 & 10.16 & 11.15 & 12.61 & 12.73 & 10.82 & 10.19 & 10.31 \\
\hline $\mathrm{MnO}$ & 0.20 & 0.20 & 0.19 & 0.20 & 0.19 & 0.20 & 0.19 & 0.20 & 0.20 & 0.20 & 0.17 & 0.16 & 0.16 \\
\hline $\mathrm{MgO}$ & 7.12 & 7.07 & 6.87 & 6.38 & 7.74 & 8.13 & 7.23 & 7.60 & 7.11 & 7.14 & 9.26 & 9.30 & 9.59 \\
\hline $\mathrm{CaO}$ & 10.48 & 10.64 & 11.19 & 9.94 & 9.85 & 12.41 & 9.14 & 10.87 & 10.07 & 10.14 & 9.89 & 10.95 & 10.69 \\
\hline $\mathrm{Na}_{2} \mathrm{O}$ & 2.62 & 2.44 & 2.41 & 3.57 & 2.59 & $\begin{array}{l}1.87 \\
\end{array}$ & 3.22 & 2.67 & $\begin{array}{l}2.83 \\
\end{array}$ & 2.50 & 2.48 & 2.64 & 2.59 \\
\hline $\mathrm{K}_{2} \mathrm{O}$ & 0.45 & 0.49 & 0.45 & 0.26 & 0.16 & 0.15 & 0.94 & 0.63 & 0.42 & 0.45 & 0.48 & 0.39 & 0.39 \\
\hline $\mathrm{P}_{2} \mathrm{O}_{5}$ & 0.11 & 0.11 & 0.11 & 0.11 & 0.10 & 0.11 & 0.11 & 0.12 & 0.12 & 0.12 & 0.08 & 0.08 & 0.08 \\
\hline LOI & 2.16 & 2.36 & 1.87 & 1.56 & 2.78 & 2.33 & 4.00 & 7.08 & 2.03 & 2.00 & 2.69 & 2.40 & 2.47 \\
\hline Total & 99.49 & 99.56 & 99.40 & 99.78 & 99.01 & 100.12 & 99.23 & 99.31 & 99.53 & 99.71 & 100.01 & 99.93 & 99.39 \\
\hline & & & & & & & & & & & & & \\
\hline Sc & 42.17 & 44.26 & 39.68 & 45.11 & 45.16 & 42.44 & 39.92 & 44.98 & 43.74 & 44.32 & 41.70 & 40.58 & 41.68 \\
\hline $\mathrm{V}$ & 295.05 & 308.72 & 286.91 & 312.98 & 279.85 & 297.17 & 271.31 & 285.56 & 302.59 & 301.54 & 245.20 & 240.11 & 237.68 \\
\hline $\mathrm{Cr}$ & 89.02 & 95.05 & 78.74 & 82.57 & 286.28 & 312.40 & 106.06 & 125.21 & 100.13 & 94.33 & 382.24 & 417.99 & 429.84 \\
\hline Co & 50.65 & 51.01 & 45.92 & 48.81 & 49.98 & 51.48 & 42.54 & 44.55 & 46.02 & 43.02 & 46.15 & 46.55 & 46.88 \\
\hline $\mathrm{Ni}$ & 49.89 & 52.52 & 41.69 & 58.01 & 78.24 & 89.02 & 44.07 & 47.26 & 55.63 & 51.81 & 106.66 & 114.10 & 112.09 \\
\hline $\mathrm{Cu}$ & 109.74 & 104.56 & 107.74 & 106.50 & 154.88 & 65.96 & 6.36 & 5.09 & 68.64 & 72.63 & 95.73 & 107.63 & 104.99 \\
\hline $\mathrm{Zn}$ & 72.40 & 74.01 & 68.48 & 72.97 & 66.28 & 55.21 & 58.86 & 64.76 & 62.80 & 56.96 & 49.51 & 42.84 & 41.31 \\
\hline $\mathrm{Ga}$ & 13.44 & 14.58 & 13.52 & 14.12 & 11.98 & 14.43 & 11.68 & 12.87 & 14.90 & 14.41 & 12.58 & 11.02 & 10.76 \\
\hline $\mathrm{Rb}$ & $\begin{array}{l}8.67 \\
\end{array}$ & 9.48 & 9.17 & 4.27 & 2.19 & 2.13 & 22.34 & 13.86 & 8.96 & 9.56 & 10.93 & 7.07 & 7.79 \\
\hline $\mathrm{Sr}$ & 206.07 & 205.43 & 189.59 & 248.04 & 172.73 & 216.11 & 200.92 & 197.05 & 172.45 & 167.49 & 210.16 & 156.79 & 153.26 \\
\hline $\mathrm{Y}$ & 21.52 & 21.43 & 19.05 & 21.78 & 17.08 & 19.21 & 19.98 & 21.77 & 23.56 & 23.01 & 16.10 & 15.82 & 15.45 \\
\hline $\mathrm{Zr}$ & 64.16 & 63.44 & 59.55 & 59.72 & 55.56 & 56.59 & 62.42 & 70.60 & 74.56 & 73.31 & 45.09 & 43.87 & 42.24 \\
\hline $\mathrm{Nb}$ & 3.14 & 3.38 & 3.24 & 3.02 & 2.84 & 2.83 & 3.01 & 3.40 & 3.19 & 3.58 & 2.23 & 2.27 & 2.33 \\
\hline $\mathrm{Ba}$ & 91.90 & 91.01 & 76.49 & 74.57 & 51.21 & 38.41 & 178.81 & 141.77 & 73.90 & 76.59 & 102.61 & 125.24 & 110.75 \\
\hline $\mathrm{La}$ & 4.65 & 4.68 & 4.53 & 4.53 & 3.73 & 3.63 & 4.27 & 5.35 & 4.94 & 4.67 & 2.83 & 3.23 & 3.18 \\
\hline Ce & 11.69 & 12.43 & 12.04 & 11.43 & 9.72 & 9.56 & 11.19 & 13.13 & 12.82 & 12.43 & 7.99 & 8.24 & 8.44 \\
\hline $\operatorname{Pr}$ & 1.77 & 1.83 & 1.85 & 1.65 & 1.44 & 1.42 & 1.69 & 2.01 & 1.94 & 1.91 & 1.13 & 1.26 & 1.27 \\
\hline $\mathrm{Nd}$ & 9.71 & 9.40 & 8.99 & 8.43 & 7.37 & 7.50 & 7.97 & 9.55 & 9.86 & 9.67 & 5.94 & 6.50 & 6.54 \\
\hline Sm & 2.79 & 2.71 & 2.45 & 2.59 & 2.14 & 2.70 & 2.57 & 3.33 & 3.21 & 2.95 & 2.05 & 2.21 & 2.43 \\
\hline $\mathrm{Eu}$ & 1.00 & 0.98 & 0.94 & 1.02 & 0.93 & 0.93 & 0.56 & 0.88 & 0.87 & 0.86 & 0.43 & 0.80 & 0.81 \\
\hline $\mathrm{Gd}$ & 3.50 & 3.39 & 3.18 & 3.38 & 3.24 & 3.05 & 3.49 & 3.61 & 4.05 & 3.97 & 2.73 & 2.89 & 2.92 \\
\hline $\mathrm{Tb}$ & 0.59 & 0.60 & 0.59 & 0.59 & 0.52 & 0.52 & 0.55 & 0.64 & 0.69 & 0.62 & 0.46 & 0.44 & 0.41 \\
\hline Dy & 4.21 & 4.14 & 3.48 & 3.70 & 2.82 & 3.53 & 3.34 & 4.33 & 4.09 & 4.12 & 2.60 & 2.69 & 2.86 \\
\hline Ho & 0.79 & 0.86 & 0.73 & 0.82 & 0.73 & 0.70 & 0.72 & 0.89 & 0.90 & 0.80 & 0.59 & 0.67 & 0.61 \\
\hline $\mathrm{Er}$ & 2.35 & 2.54 & 2.27 & 2.58 & 1.92 & 1.90 & 2.03 & 2.56 & 2.56 & 2.64 & 1.86 & 1.83 & 1.64 \\
\hline $\mathrm{Tm}$ & 0.36 & 0.33 & 0.31 & 0.32 & 0.32 & 0.32 & 0.27 & 0.36 & 0.42 & 0.32 & 0.25 & 0.23 & 0.26 \\
\hline $\mathrm{Yb}$ & 2.27 & 2.46 & 1.91 & 2.49 & 1.97 & 2.19 & 2.07 & 2.49 & 2.29 & 2.27 & 1.88 & 2.03 & 1.53 \\
\hline $\mathrm{Lu}$ & 0.34 & 0.31 & 0.28 & 0.33 & 0.29 & 0.30 & 0.29 & 0.33 & 0.32 & 0.38 & 0.21 & 0.23 & 0.27 \\
\hline Hf & 1.60 & 1.82 & 1.76 & 1.77 & 1.56 & 1.36 & 1.63 & 2.03 & 2.08 & 2.02 & 1.22 & 1.02 & 1.22 \\
\hline $\mathrm{Ta}$ & 0.26 & 0.27 & 0.26 & 0.26 & n.d. & 0.17 & 0.18 & 0.24 & 0.24 & 0.23 & 0.18 & 0.15 & 0.15 \\
\hline $\mathrm{Pb}$ & 1.06 & 1.05 & 1.07 & 1.63 & 0.55 & 0.82 & 0.86 & 1.01 & 0.29 & 0.25 & 0.21 & 0.29 & 0.33 \\
\hline Th & 0.50 & 0.52 & 0.57 & 0.50 & 0.32 & 0.30 & 0.50 & 0.58 & 0.51 & 0.60 & 0.24 & 0.24 & 0.30 \\
\hline $\mathrm{U}$ & 0.15 & 0.16 & 0.13 & 0.10 & n.d. & 0.04 & 0.19 & 0.21 & 0.17 & 0.17 & 0.10 & 0.04 & 0.07 \\
\hline
\end{tabular}

n.d: not detected.

The MORB-normalized patterns of the basalts (Fig. 5A) show variable enrichment in LILE, variable depletion in $\mathrm{Zr}$, Hf, Sm, Ti, Y and $\mathrm{Yb}$, and small negative $\mathrm{Nb}$ anomaly, reflecting subduction component in their magma source. Ta and Ce range from slightly enriched to slightly depleted relative to N-MORB. The chondrite-normalized REE patterns of the basalts (Fig. 5B) display slight enrichment in LREE relative to $\operatorname{HREE}\left(\mathrm{La} / \mathrm{Yb}_{\mathrm{n}}=1.08-1.70\right)$. Eu anomalies range from slightly positive to markedly negative $\left(\mathrm{Eu} / \mathrm{Eu}^{*}=1.08-0.56\right)$. The slightly positive $\mathrm{Eu}$ anomalies suggest some plagioclase accumulation, while the strongly negative anomalies reflect significant plagioclase fractionation.

\section{DISCUSSION}

\section{Tectonic setting: back-arc or nascent island arc?}

The volcanic rocks of the study area were mapped as island arc volcanics (Bakhit, 1994; Basta et al., 1996). The chemical characteristics of the pyroxenes of Wadi Atalla El-Murr basalts resemble those of pyroxenes of ocean-floor and island arc basalts and are similar to those of pyroxenes of basalts erupted along modern back-arcs such as Lau Basin (Fig. 3C and D). Moreover, the MORBnormalized spider diagrams of the basalts (Fig. 5A) display small Nb trough and LILE enrichment, implying subduction component in the mantle source of the parental magma produced Wadi Atalla El- 
Murr basalts. However, plotting the analyzed basalts on different tectonomagmatic discrimination diagrams (Fig. 6A-D) suggests that they were erupted on ocean floor rather than in island arc setting.
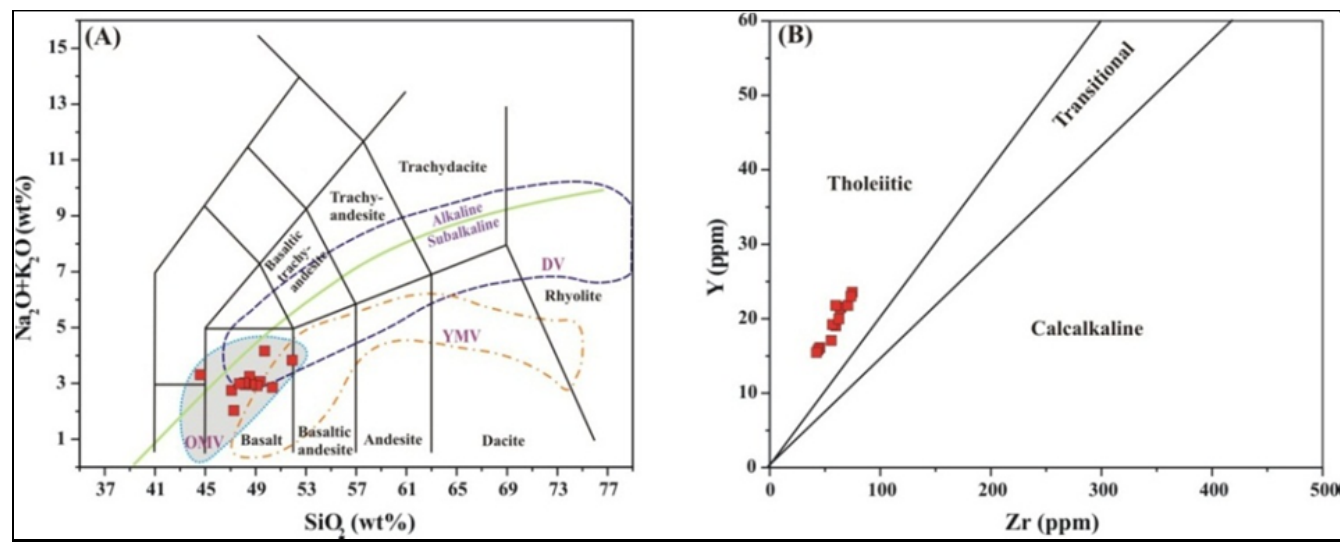

Fig. 4. Classification and magma type of Wadi Atalla El-Murr mafic volcanic rocks. (A) $\mathrm{SiO}_{2}$ versus $\mathrm{Na}_{2} \mathrm{O}+\mathrm{K}_{2} \mathrm{O}$ diagram (after Le Bas et al., 1986), (B) Zr-Y diagram (after Barrett and MacLean, 1994). The fields of Older metavolcanics (OMV), Younger metavolcanics (YMV) and Dokhan volcanics (DV) are adopted from Eliwa et al. (2006) and references therein. The dividing line between subalkaline and alkaline fields after Irvine and Baragar (1971).

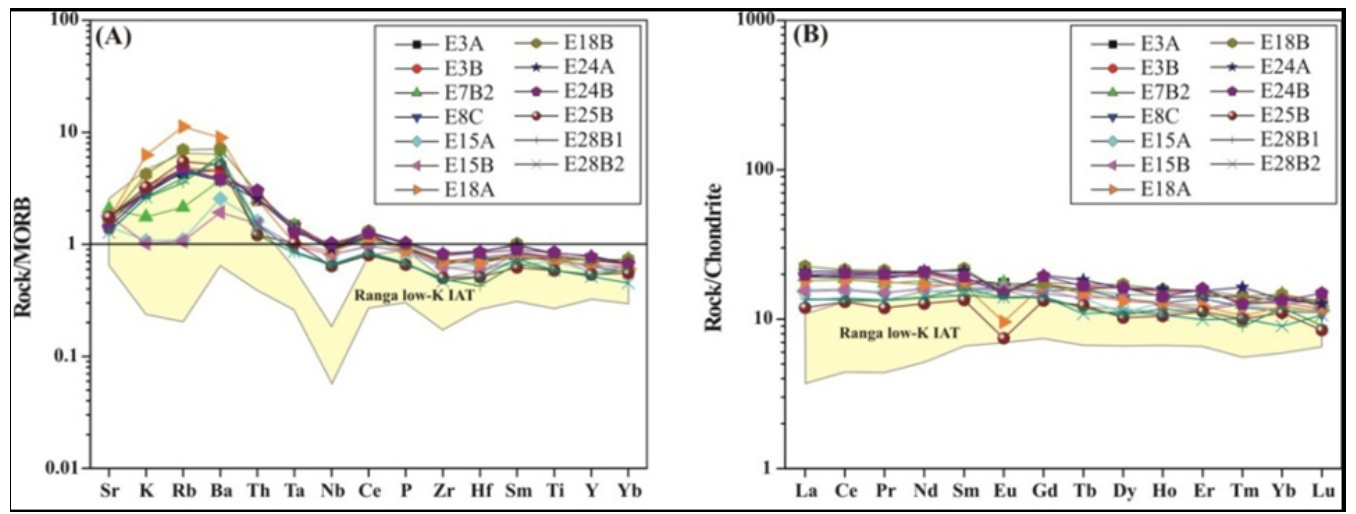

Fig. 5. (A) MORB-normalized spider diagrams and (B) Chondrite-normalized REE patterns for Wadi Atalla ElMurr metabasalts. MORB normalization values after Pearce (1983); Chondrite normalization values after Sun and McDonough (1989). Data of Wadi Ranga IAT after Maurice et al. (2012).

Tholeiitic basalts like those of Wadi Atalla El-Murr can be generated along spreading centers or in intra-oceanic island arc setting (Wilson, 1989). To evaluate the possibility of generation of Wadi Atalla El-Murr Neoproterozoic basalts in nascent island arc, the geochemical features of these basalts have been compared with those of Wadi Ranga Neoproterozoic basalts which were extruded in nascent intra-oceanic island arc setting (Maurice et al., 2012). Although they plot close to those of Wadi Atalla El-Murr basalts, the pyroxenes of Wadi Ranga and Wadi Atalla El-Murr tholeiites do not overlap (Fig. 3). On the different tectonomagmatic discrimination diagrams, it is evident that Wadi Ranga IAT and Wadi Atalla El-Murr basalts occupy island arc and MORB or BABB fields (Fig. 6), respectively. Moreover, the spider diagrams of these tholeiitic basalts are different, where the patterns of Wadi Ranga IAT display stronger depletion in HFSE and very marked Nb trough compared with those of the basalts of Wadi Atalla El-Murr (Fig. 5A). The Wadi Atalla El-Murr basalts have REE abundances higher than those of Wadi Ranga IAT (Fig. 5B), and their chondrite-normalized REE patterns display slight LREE-enrichment $\left(\mathrm{La} / \mathrm{Yb}_{\mathrm{n}}=1.08-1.70\right)$ while those of Wadi Ranga ITA are characterized mostly by LREE-depletion $\left(\mathrm{La} / \mathrm{Yb}_{\mathrm{n}}=0.49-1.03\right)$. In short, the geochemical features of Wadi Ranga IAT and Wadi Atalla El-Murr basalt are not alike, ruling out the extrusion of Wadi Atalla El-Murr basalts in nascent intra-oceanic island arc.

The mixed MORB/IAT geochemical and mineral chemical characteristics of the Wadi Atalla ElMurr basalts resemble those of basalts generated in modern back-arc environments such as Scotia Sea, Lau Basin and Mariana Trough (e.g. Tarney et al., 1981; Saunders and Tarney, 1984; Pearce et al., 1995; 2005; Fretzdorff et al., 2002; Keller et al., 2008), and are best explained by generation of the parental melts of these basalts along spreading center located close to subduction zone, i.e. along Neoproterozoic back-arc spreading center. Additionally, plotting the analyzed samples on the Ti-V 
(Shervais, 1982) and La-Y-Nb (Cabanis and Lecolle, 1989) diagrams (Fig. 6C and D), supports backarc environment for the generation of Wadi Atalla El-Murr basalts.
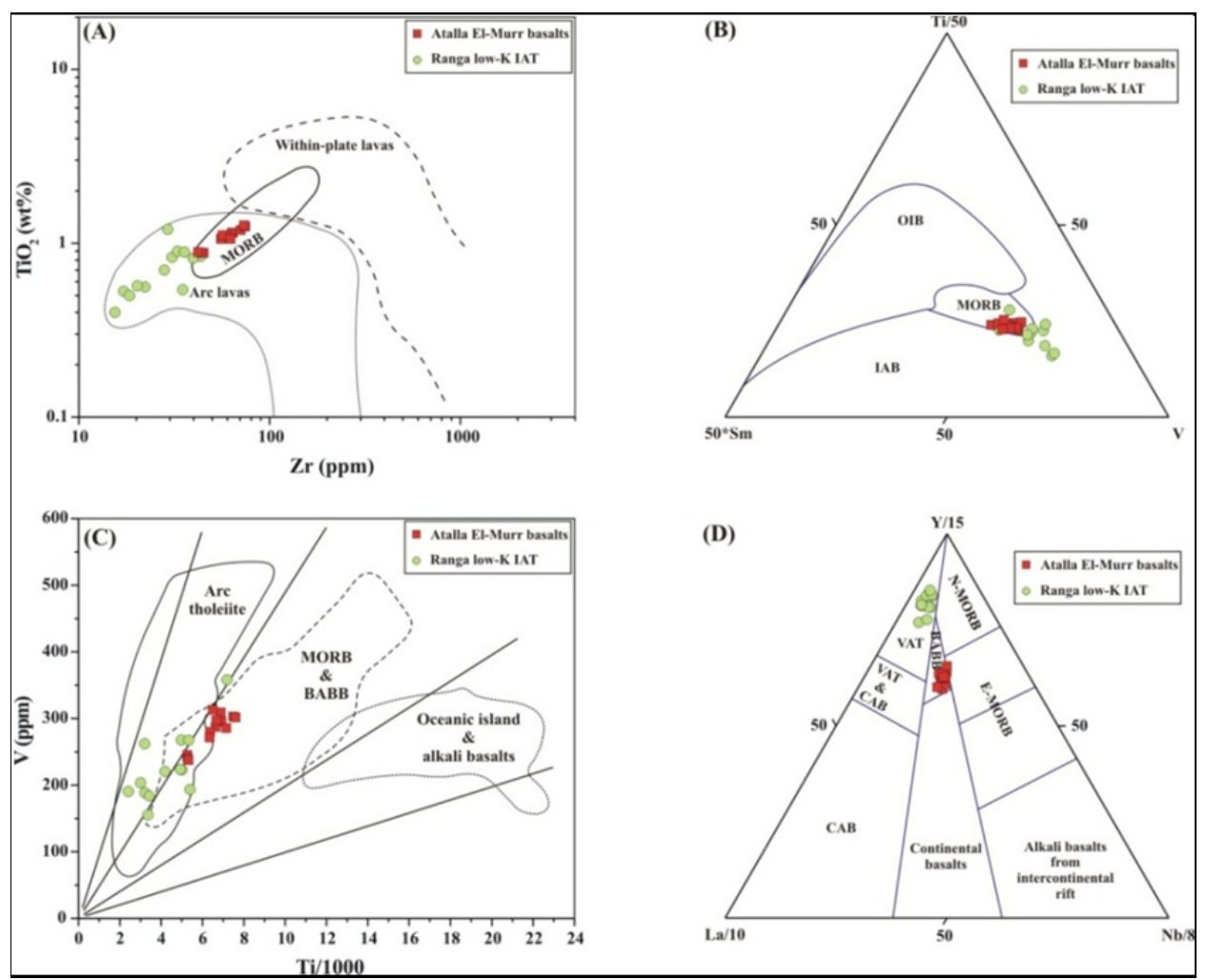

Fig. 6. Tectonic setting of Wadi Atalla El-Murr metabasalts. (A) $\mathrm{Zr}-\mathrm{TiO}_{2}$ diagram (after Pearce, 1982), (B) Sm-Ti-V diagram (after Vermeesch, 2006), (C) Ti-V diagram (after Shervais, 1982), (D) La-Y-Nb diagram (after Cabanis and Lecolle, 1989). Data of Wadi Ranga IAT after Maurice et al. (2012).

\section{Petrogenesis}

The depletion of most HFSE in Wadi Atalla El-Murr basalts compared with N-MORB (Fig. 5A) indicates that the mantle source of the parental magmas of these basalts was more depleted than NMORB mantle source. Additionally, Most of the trace elements abundances of the Wadi Atalla ElMurr basalts are lower than those of the Neoproterozoic N-MORB of Gerf ophiolite (Fig. 7), supporting mantle source more depleted than N-MORB mantle source. The $\mathrm{Nb}$ and $\mathrm{Zr}-\mathrm{Hf}$ troughs characteristic for the trace elements patterns of Wadi Atalla El-Murr basalts compared with the Neoproterozoic N-MORB of Gerf ophiolite (Fig. 7), indicate SSZ mantle source for these basalts (Pearce, 2014). On the $\mathrm{Th} / \mathrm{Yb}$ vs Nb/Yb diagram (Pearce, 2014), the Neoproterozoic Wadi Atalla ElMurr basalts plot close to or above the MORB-OIB array (Fig. 8A), suggesting subduction component in the mantle source of their parental magma. This is supported by LILE-enrichment relative to MORB (Fig. 5A) and the slight enrichment in LREE relative to HREE $\left(\mathrm{La} / \mathrm{Yb}_{\mathrm{n}}=1.08-1.70\right)$ of Wadi Atalla El-Murr basalts (Fig. 5B). Although the analyzed samples of Wadi Atalla El-Murr plot within the mantle array on the $\mathrm{La} / \mathrm{Yb}$ vs $\mathrm{Nb} / \mathrm{Yb}$ diagram (Fig. 8B), their $\mathrm{La} / \mathrm{Yb}$ values are higher than $\mathrm{N}$ MORB value implying contribution of small amount of LREE through subduction component. The enrichment of Wadi Atalla El-Murr metabasalts in fluid mobile elements compared with MORB (Fig. 5A) suggest subducted fluid component in their mantle source. On the other hand, the higher Th abundances in these metabasalts compared with N-MORB (Figs. 7 and 8A) can be attributed to subducted sediments (Plank, 2005; Tian et al., 2011) during deep subduction (Pearce and Stern, 2006). However, the subduction component was not significant as indicated by slight LREE-enrichment on their REE patterns (Fig. 5B). The variable Mg\# (52.7-64.8) of Wadi Atalla El-Murr basalts together with $\mathrm{Eu}$ anomaly values ranging from slightly positive to markedly negative $\left(\mathrm{Eu} / \mathrm{Eu}^{*}=1.08-0.56\right)$ indicate that some of these basalts do not represent primitive basaltic melts and their compositions have been modified by fractionation before eruption. 
Fig. 7. N-MORB-normalized trace elements of Wadi Atalla El-Murr and the average of Neoproterozoic N-MORB of Gerf ophiolite. Normalization values after Sun and McDonough (1989).
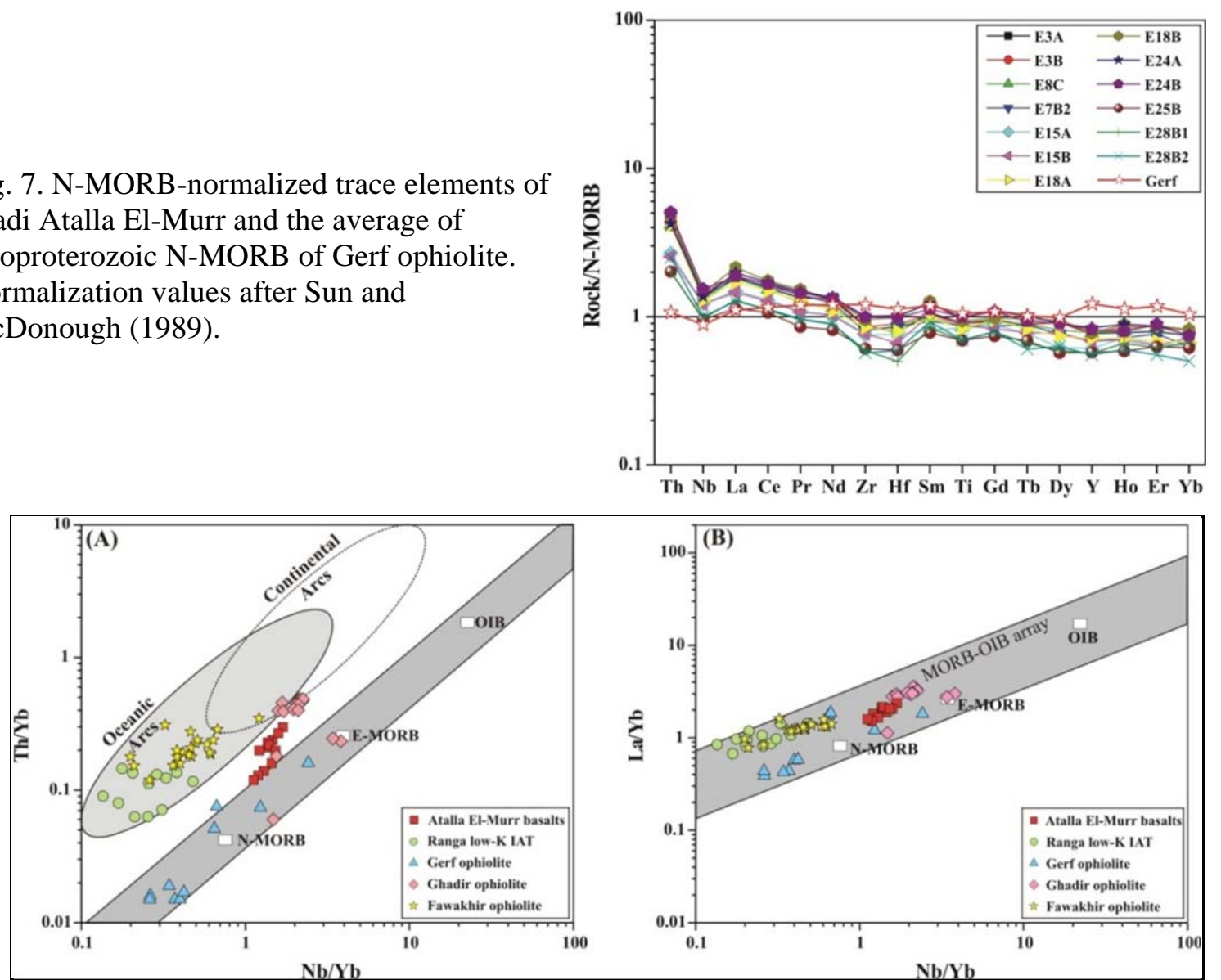

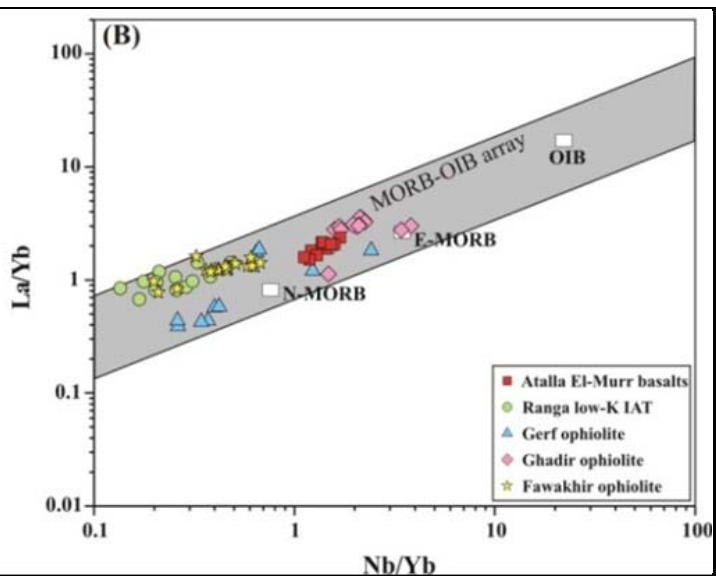

Fig. 8: Evaluation of subduction component in Wadi Atalla El-Murr basalts and other Egyptian ophiolite metavolcanics, the Neoproterozoic Wadi Ranga IAT is shown for comparison. (A) $\mathrm{Th} / \mathrm{Yb}$ vs $\mathrm{Nb} / \mathrm{Yb}$ diagram (after Pearce, 2014), (B) La/Yb vs Nb/Yb diagram, the MORB-OIB array after Green (2006). Data sources: Wadi Atalla El-Murr ophiolite metavolcanics (present study), Gerf ophiolite (Zimmer et al., 1995); Wadi Ghadir ophiolite (Basta et al., 2011); Fawakhir ophiolite (Abd El-Rahman et al., 2009b); Wadi Ranga IAT (Maurice et al., 2012).

\section{Back-arc basin maturity}

Although they are formed along spreading centers, back-arc basin basalts (BABB) and MORB may differ in geochemical features (Fryer et al., 1981; Tarney et al., 1981). Studies of volcanic rocks of modern back-arcs such as Lau Basin, Mariana Trough and East Scotia Sea showed that the composition of basalts varied spatially along the different segments of back-arc ridge from MORBlike to arc-like (Tarney et al., 1981; Pearce et al., 1995, 2005; Keller et al., 2008). Such variations have been attributed to the maturity of adjacent subduction zone, the proximity of spreading centers to subducted slab, the maturity or evolution of back-arc basin and the differences in the degree of mantle depletion (Tarney et al., 1981; Saunders and Tarney, 1984; Pearce et al., 1995; 2005; Fretzdorff et al., 2002; Pearce and Stern, 2006; Keller et al., 2008; Tian et al., 2011). Back-arc basins formed during the earliest stages of subduction erupt volcanics with MORB-like composition, whereas basalts formed during the early stages of back-arc spreading in mature subduction zone have arc-like composition (Tarney et al., 1981; Saunders and Tarney, 1984; Keller et al., 2008). Back-arc spreading centers present close to arc erupt basalts with arc signature, while those located far from trench produce MORB-like volcanics (e.g. Pearce et al., 1995). The subduction signature in back-arc magmas gradually decreases with increasing the distance from the arc. Moreover, the composition of the subduction component of basalts of Lau Basin varies with the proximity of the spreading center to the arc (Pearce et al., 1995).

The N-MORB-normalized trace elements patterns of Wadi Atalla El-Murr basalts (Fig. 7) display $\mathrm{Nb}$ troughs characteristic for SSZ ridge settings, and small Zr-Hf troughs characteristic for BABB erupted along spreading center adjacent to arcs (Pearce, 2014). On the $\mathrm{Th} / \mathrm{Yb}$ vs $\mathrm{Nb} / \mathrm{Yb}$ diagram (Pearce, 2014), the Neoproterozoic back-arc basalts of Wadi Atalla El-Murr plot close to or above the MORB-OIB array (Fig. 8A), suggesting subduction component in their mantle source. Plotting the 
analyzed basalts on the $\mathrm{Th}_{\mathrm{N}}-\mathrm{Nb}_{\mathrm{N}}$ diagram of Saccani (2015) shows that most of the samples lie along the boundary between the fields of immature and mature intra-oceanic back-arcs (Fig. 9A), suggesting eruption of these volcanics during immature-mature transition stage or during the earliest mature stage of Neoproterozoic intra-oceanic back-arc basin. This conclusion is supported by the fact that some of the analyzed samples plot in the overlap area between the arc and MORB/BABB fields on the Ti-V diagram (Fig. 6C) of Shervais (1982).

\section{Implication for the composition, settings and classification of ANS ophiolites}

The ophiolite metavolcanics of Egypt have compositions ranging from basalts to basaltic andesites with occasional andesites (e.g. El-Sharkawy and El-Bayoumi, 1979; Basta, 1983; Abd Rl-Rahman et al., 2009a \& b; Basta et al., 2011). To evaluate the compositional variations of the ophiolite metavolcanics of the Eastern Desert, the composition of samples from the ophiolites of Gerf (Zimmer et al., 1995), Wadi Ghadir (Basta et al., 2011) and Fawakhir (Abd Rl-Rahman et al., 2009b) has been plotted on the $\mathrm{Th}_{\mathrm{N}}-\mathrm{Nb}_{\mathrm{N}}$ diagram of Saccani (2015). This diagram (Fig. 9B) showed that the metavolcanic rocks of Egyptian ophiolites comprise MORB and BABB compositions, and the MORB composition varies from depleted through normal to enriched. Such variation in the MORB composition implies Neoproterozoic mantle heterogeneity (Khedr and Arai, 2016).

The ophiolites are classified into subduction-unrelated and subduction-related types (Dilek and Furnes, 2014). The former comprise continental margin, mid-ocean ridge and plume-types, while the latter include fore/back-arc suprasubduction zone and volcanic arc types. Based on the geochemical affinities of the ophiolite metavolcanic rocks, Abd El-Rahman et al. (2012) classified the ophiolites of the Central Eastern Desert of Egypt into fore-arc and back-arc types. They suggested that the fore-arc and back-arc ophiolites are preserved in the western and eastern parts of the Central Eastern Desert, respectively. Recently, El Bahariya (2018) divided the ophiolites of the Central Eastern Desert of Egypt into MORB or BABB and SSZ types, although BABB ophiolite is considered sub-type of SSZ ophiolites (Dilek and Furnes, 2014). He proposed that these ophiolites are temporally and spatially unrelated and their setting has been changed with time from MORB to SSZ.

Figures $8 \mathrm{~A}$ and $9 \mathrm{~A}$ showed that the tectonic settings of the metavolcanics of the famous ophiolites of the Eastern Desert comprise subduction-unrelated (Gerf) and subduction-related (present study, Wadi Ghadir, Fawakhir) types. In the subduction-related types, the magnitude of subduction input to the mantle source of the Neoproterozoic ophiolite metavolcanics of the Eastern Desert varied from small (present study) to significant (Fawakhir), implying generation at variable distances from adjacent arcs during different stages of evolutions of Neoproterozoic SSZ systems.
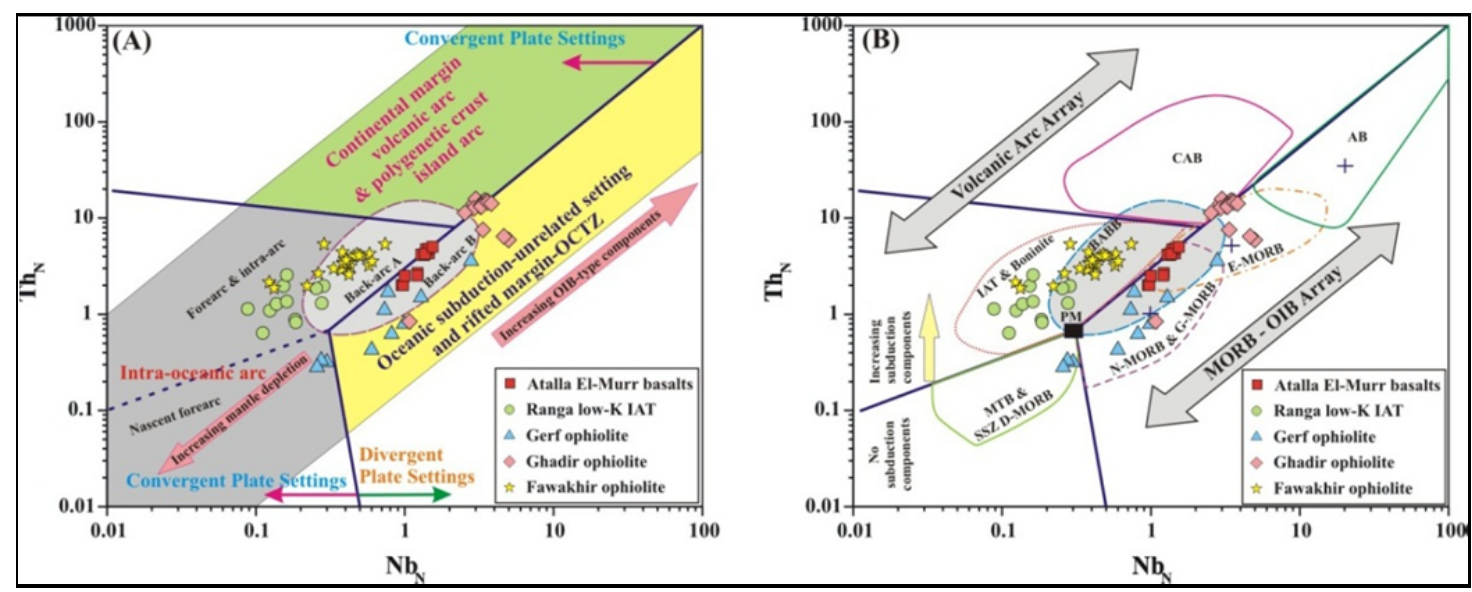

Fig. 9: $\mathrm{Th}_{\mathrm{N}}$ versus $\mathrm{Nb}_{\mathrm{N}}$ diagram (after Saccani, 2015) to evaluate (A) the tectonic settings and (B) the compositions of Egyptian ophiolite metavolcanics. Th and $\mathrm{Nb}$ are normalized to N-MORB values of Sun and McDonough (1989). Back-arc A, BABB with subduction component; Back-arc B, BABB with no subduction input. Data sources as in Fig. 8.

\section{CONCLUSIONS}

1- The Wadi Atalla El-Murr metabasalts are composed of pyroxene and plagioclase with texture varying from very fine-grained through vitrophyre to porphyritic which reflects variable degrees of cooling from quenching to slower cooling rate. 
2- The pyroxenes are represented by augites while the plagioclases range in composition from labradorite $(\mathrm{An}=\sim 73 \%)$ to albite $(\mathrm{An}=0.61 \%)$, reflecting seafloor hydrothermal alteration and metamorphism.

3- The analyzed pyroxenes are similar to those crystallize from subalkaline magmas and have chemical characteristics overlapping those of the pyroxenes of island arc and ocean-floor basalts.

4- The metabasalts of Wadi Atalla El-Murr have low $\mathrm{K}_{2} \mathrm{O}$ contents $(<1 \mathrm{wt} \%)$ and tholeiitic affinity, similar to oceanic tholeiites produced by partial melting of depleted mantle sources.

5- The Wadi Atalla El-Murr basalts generally occupy the MORB or BABB field on conventional tectonomagmatic discrimination diagrams. However, the REE patterns of the metabasalts display slight enrichment in LREE relative to $\operatorname{HREE}\left(\mathrm{La} / \mathrm{Yb}_{\mathrm{n}}=1.08-1.70\right)$ and the MORB-normalized spider diagrams show variable LILE enrichment, variable depletion in most HFSE and small negative $\mathrm{Nb}$ anomaly, reflecting subduction component in a depleted mantle source.

6- The mixed MORB/IAT geochemical characteristics of the Wadi Atalla El-Murr basalts resemble those of basalts generated in modern back-arc environments affected by minor subduction component.

7- The small arc geochemical signature in the Wadi Atalla El-Murr basalts is consistent with eruption in Neoproterozoic mature back-arc basin and rules out the possibility of formation of these tholeiites in Neoproterozoic nascent intra-oceanic island arc environment.

8- The compositional characteristics of Wadi Atalla El-Murr BABB are consistent with eruption along spreading center at intermediate distance from the adjacent arc during immature-mature transition stage or during the earliest mature stage of Neoproterozoic intra-oceanic back-arc basin.

9- The metavolcanic rocks of Egyptian ophiolites comprise MORB and BABB compositions, and the MORB composition varies from depleted through normal to enriched.

10- The ophiolites of the Eastern Desert comprise subduction-unrelated (Gerf) and subduction-related (present study, Wadi Ghadir, Fawakhir) types. Variable magnitude of subduction signature in the metavolcanics of the Egyptian SSZ ophiolites reflects generation at variable stages of evolution of Neoproterozoic SSZ systems.

\section{ACKNOWLEDGEMENTS}

The author expresses his gratitude to Prof. Dr. Bottros R. Bakhit, Beni-Suef University, for the electron microprobe and geochemical analyses conducted at ETH-Zürich, Switzerland. Prof. Dr. Fawzy Basta, Cairo University, is acknowledged for fruitful discussions and reading an earlier version of the manuscript. The author whishes to thank Prof. Dr. Paul Asimow, Caltech, USA, for reading part of the manuscript, and Prof. Dr. Baher El-Kaliouby and Dr. Nahla Abd El-Ghaffar for editorial handling.

\section{REFERENCES}

Abdel-Rahman, A. M. (1996): Pan-African volcanism: petrology and geochemistry of the Dokhan Volcanic suite in the northern Nubian Shield. Geol. Mag., 133, 17-31.

Abd El-Rahman, Y., Polat, A., Dilek, Y., Fryer, B., El-Sharkawy, M. and Sakran, S. (2009a): Geochemistry and tectonic evolution of the Neoproterozoic Wadi Ghadir Ophiolite, Eastern Desert, Egypt. Lithos, 113 (1-2), 158-178.

Abd El-Rahman, Y., Polat, A., Dilek, Y., Fryer, B., El-Sharkawy, M. and Sakran, S. (2009b): Geochemistry and tectonic evolution of the Neoproterozoic incipient arc-forearc crust in the Fawakhir area, Central Eastern Desert of Egypt. Precamb. Res., 175, 116-134.

Abd El-Rahman, Y., Polat, A., Dilek, Y., Kusky, T.M., El-Sharkawi, M. and Said, A. (2012): Cryogenian ophiolite tectonics and metallogeny of the Central Eastern Desert of Egypt. Inter. Geol. Rev., 54 (16), 1870-1884.

Abdel-Karim, A. M., Ali, S., El-Awady, A., Elwan, W., Khedr, M. and Akihiro, T. (2019): Mineral and bulk-rock chemistry of Shadli bimodal metavolcanics from Eastern Desert of Egypt: Implication for tectonomagmatic setting and Neoproterozoic continental growth in the ArabianNubian Shield. Lithos, 338-339, 204-217.

Ali, K. A., Stern, R. J., Manton, W. I., Kimura, J.-I. and Khamees, H. A. (2009): Geochemistry, Nd isotopes and U-Pb SHRIMP zircon dating of Neoproterozoic volcanic rocks from the Central Eastern Desert of Egypt: New insights into the 750 Ma crust-forming event. Precamb. Res., 171, $1-22$. 
Maurice, A. E.

Bakhit, B. R. (1994): Gold mineralization in some occurrences of the Eastern Desert, Egypt. M.Sc Thesis, Cairo Univ., 214p.

Barrett, T. J., MacLean, W. H., (1994): Chemostratigraphy and hydrothermal alteration in exploration for VHMS deposits in greenstone and younger volcanic rocks. In: Lentz, D. R. (Ed.), Alteration and Alteration Processes Associated with Ore-Forming Systems. Geological Association of Canada, Short Course Notes, vol. 11, 433-467.

Basta, E. Z., Kotb, H. and Awadalla, M. F. (1980): Petrochemical and geochemical characteristics of the Dokhan Formation at the type locality, Jabal Dokhan, Eastern Desert, Egypt. Inst. Appl. Geol. Jeddah Bull., 3, 121-140.

Basta, F. F., (1983): Geology and geochemistry of the ophiolitic mélange and other rock units in the area around and west of Gabal Ghadir, Eastern Desert, Egypt. Ph.D. thesis, Cairo University, 137p.

Basta, F. F., Maurice, A. E., Bakhit, B. R., Ali, K. A. and Manton, W. I. (2011): Neoproterozoic Contaminated MORB of Wadi Ghadir Ophiolite, NE Africa: Geochemical and Nd and Sr isotopic Constraints. J. Afr. Earth Sci., 59, 227-242.

Basta, F. F., Maurice, A. E., Bakhit, B. R., Azer, M. K. and El-Sobky, A. F. (2017): Intrusive rocks of the Wadi Hamad area, North Eastern Desert, Egypt: change of magma composition with maturity of Neoproterozoic continental island arc and the role of collisional plutonism in the differentiation of arc crust. Lithos, 288-289, 248-263.

Basta, F. F., Takla, M. A. and Bakhit, B. R. (1996): Gold mineralization of Atalla and Semna areas, Eastern Desert, Egypt. Third Inter. Conf. Geol. Arab World, 63-86.

Beccaluva, L., Macciotta, G., Piccardo, G. B. and Zeda, O. (1989): Clinopyroxene of ophiolite basalts as petrogenetic indicator. Chem. Geol., 77, 165-182.

Cabanis, B. and Lecolle, M., (1989): Le diagramme La/10-Y/15-Nb/8: un outil pour la discrimination des séries volcaniques et la mise en évidence des procésses de mélange et/ou de contamination crustale. C. R. Acad. Sci., 2, 2023-2029.

Dilek, Y. and Furnes, H. (2014): Ophiolites and their origins. Elements 10, 93-100.

El Bahariya, G. A. (2018): Classification of the Neoproterozoic ophiolites of the Central Eastern Desert, Egypt based on field geological characteristics and mode of occurrence. Arab. J. Geosci., $11,313$.

Eliwa, H. A., Kimura, J.-I. and Itaya, T. (2006): Late Neoproterozoic Dokhan Volcanics, North Eastern Desert, Egypt: Geochemistry and petrogenesis. Precamb. Res., 151, 31-52.

El-Sayed, M. M., Furnes, H. and Mohamed, F. H. (1999): Geochemical constraints on the tectonomagmatic evolution of the late Precambrian Fawakhir ophiolite, Central Eastern Desert, Egypt. J. Afr. Earth Sci., 29 (3), 515-533.

El-Sharkawy, M. A. and El-Bayoumi, R. (1979): The ophiolites of Wadi Ghadir area, Eastern Desert, Egypt. Ann. Geol. Surv. Egypt, 9, 125-135.

Essawy, M. A. and Abu Zeid, K. M. (1972): Atalla felsite intrusion and its neighbouring rhyolitic flows and tuffs, Eastern Desert. Ann. Geol. Surv. Egypt, 2, 271-280.

Fretzdorff, S., Livermore, R. A., Devey, C. W., Leat, P. T. and Stoffers, P. (2002): Petrogenesis of the back-arc east Scotia Ridge, South Atlantic Ocean. J. Petrol., 43, 1435-1467.

Fryer, B., Sinton, J. M. and Philpotts, J. A. (1981): Basaltic glasses from the Mariana Trough. DSDP initial reports, 60, 601-609.

Ghoneim, M.F. (1973): Geology of the area around Bir El-Kubbania, Eastern Desert, Egypt. M. Sc. thesis, Tanta Univ., Egypt.

Green, N. L. (2006): Influence of slab thermal structure on basalt source regions and melting conditions: REE and HFSE constraints on from the Garibaldi volcanic belt, northern Cascadia subduction system. Lithos, 87, 23-49.

Gribble, R. F., Stern, R. J., Newman, S., Bloomer, S. H. and O’Hearn, T. (1998): Chemical and isotopic composition of lavas from the northern Mariana Trough: Implications for magmagenesis in back-arc basins. J. Petrol., 39, 125-154.

Hirahara, Y., Kimura, J.-I., Senda, R., Miyazaki, T., Kawabata, H., Takahashi, T., Chang, Q., Vaglarov, B. S., Sato, T. and Kodaira, S. (2015): Geochemical variations in Japan Sea back-arc basin basalts formed by high- temperature adiabatic melting of mantle metasomatized by sediment subduction components, Geochem. Geophys. Geosyst., 16, 1324 - 1347, doi:10.1002/2015GC005720. 
Hawkins, J. W. and Allan, J. F. (1994): Petrologic evolution of the Lau Basin, Sites 834-839. Proc. Ocean Drill. Program Sci. Results, 135, 427-470.

Irvine, T.N. and Baragar, W.R.A., (1971): A guide to the chemical classification of the common volcanic rocks. Can. J. Earth Sci., 8, 523-548.

Keller, N. S., Arculus, R. J., Hermann, J. and Richards, S. (2008): Submarine back-arc lava with arc signature: Fonualei Spreading Center, northeast Lau Basin, Tonga. J. Geophys. Res., 113, B08S07, doi:10.1029/2007JB005451.

Khedr, M.Z. and Arai, S. (2016): Petrology of a Neoproterozoic Alaskan-type complex from the Eastern Desert of Egypt: implications for mantle heterogeneity. Lithos, 263, 15-32.

Le Bas, M. J., Le Maitre, R. W., Streckeisen, A. and Zanettin, B. (1986): A chemical classification of volcanic rocks based on the total alkali-silica diagram. J. Petrol., 27, 745-750.

Leterrier, J., Maury, R. C., Thonon, P., Girard, D. and Marchal, M. (1982): Clinopyroxene composition as a method of identification of the magmatic affinities of paleo-volcanic series. Earth Planet. Sci. Lett., 59, 139-154.

Maurice, A. E., Bakhit, B. R., Basta, F. F, Asimow, P. D., Wälle, M, Azer, M. K. and El-Sobky, A. F. (2018): The last subduction-related volcanism in the northern tip of the Arabian-Nubian Shield: A Neoproterozoic arc preceding the terminal collision of East and West Gondwana. Precamb. Res., 310, 256-277.

Maurice, A. E., Basta, F. F. and Khiamy, A. A. (2012): Neoproterozoic nascent island arc volcanism from the Nubian Shield of Egypt: magma genesis and generation of continental crust in intraoceanic arcs. Lithos, 132-133, 1-20.

Moghazi, A. M. (2003): Geochemistry and petrogenesis of a high-K calc-alkaline Dokhan Volcanic suite, South Safaga area, Egypt: the role of late Neoproterozoic crustal extension. Precamb. Res., 125, 161-178.

Morimoto, N., Fabries, J., Ferguson, A. K., Ginzburg, I. V., Ross, M., Seifert, F. A., Zussman, J., Aoki, K. and Gottardi, G. (1988): Nomenclature of pyroxenes. Mineral. Mag., 52, 535-550.

Nisbet, F. G. and Pearce, J. A. (1977): Clinopyroxene composition in mafic lavas from different tectonic settings. Contrib. Mineral. Petrol., 63, 149-160.

Pearce, J. A. (1982): Trace element characteristics of lavas from destructive plate boundaries. In: Thorpe, R. S. (Ed.), Andesites. Wiley, Chichester, 525-548.

Pearce, J. A. (1983): Role of the subcontinental lithosphere in magma genesis at active continental margins. In: Hawkesworth, C.I. and Norry, M.J. (eds.), Continental basalts and mantle xenoliths. Shiva, Nantwich, 230-249.

Pearce, J. A. (2014): Immobile element fingerprinting of ophiolites. Elements 10, 101-108.

Pearce, J. A., Stern, R. J., Bloomer, S. and Fryer, P. (2005): Geochemical mapping of the Mariana arcbasin system: implications for the nature and distribution of subduction components. Geochem. Geophys. Geosyst., 6, Q07006. doi:10.1029/2004GC000895.

Pearce, J. A., Ernewein, M., Bloomer, S. H., Parson, L. M., Murton, B. J. and Johnson, L. E. (1995): Geochemistry of Lau Basin volcanic rocks: influence of ridge segmentation and arc proximity, In Smellie, J. L. (Ed.), Volcanism Associated with Extension at Consuming Plate Margins. Geol. Soc. Spec. Publ., 81, 53-75.

Pearce, J. A. and Stern, R. J. (2006): Origin of back-arc basin magmas: trace element and isotope perspectives. Geophysical Monograph Series, 166, 63-86.

Plank, T. (2005): Constraints from thorium/lanthanum on sediment recycling at subduction zones and the evolution of continents. J. Petrol., 46, 921-944.

Polat, A., Hofmann, A.W. and Rosing, M.T. (2002): Boninite-like volcanic rocks in the 3.7-3.8 Ga Isua greenstone belt, West Greenland: Geochemical evidence for intra-oceanic subduction zone processes in the Early Earth. Chem. Geol., 184, 231-254.

Ressetar, R. and Monard, J. R. (1983): Chemical composition and tectonic setting of the Dokhan Volcanic formation, Eastern Desert, Egypt. J. Afr. Earth Sci., 1, 103-112.

Saccani, E. (2015): A new method of discriminating different types of post-Archean ophiolitic basalts and their tectonic significance using Th-Nb and Ce-Dy-Yb systematics. Geosci. Front., 6, 481-501.

Saunders, A. D. and Tarney, J. (1984): Geochemical characteristics of basaltic volcanism within backarc basins. Geol. Soc. Lond. Spec. Publ., 17, 59-76.

Shervais, J. W. (1982): Ti-V plots and the petrogenesis of modern and ophiolitic lavas. Earth Planet. Sci. Lett., 59, 101-118. 
Shinjo, R., Chung, S.-L., Kato, Y. and Kimura, M. (1999): Geochemical and Sr-Nd isotopic characteristics of volcanic rocks from Okinawa Trough and RyuKyu Arc: implications for the evolution of a young, intracontinental back arc basin. J. Geophys. Res., 104 (B5), 10591-10608.

Sinton, J. M., Ford, L. L., Chappell, B. and McCulloch, M. T. (2003): Magma genesis and mantle heterogeneity in the Manus Back-Arc Basin, Papua New Guinea. J. Petrol., 44, 159-195.

Stern, R. J. (1981): Petrogenesis and tectonic setting of Late Precambrian ensimatic volcanic rocks, central Eastern Desert of Egypt. Precamb. Res., 16, 195-230.

Sun, S. S. and McDonough, W. F. (1989): Chemical and isotope systematics of oceanic basalts, implications for mantle composition and processes. In Saunders, A.D. and Norry, M.J. (Eds.), Magmatism in ocean basins. Geol. Soc. Lond. Spec. Publ., 42, 313-345.

Taman, Z. (1996): Geology and mineralization of Wadi Atalla area, Eastern Desert, Egypt. M.Sc thesis, Ain Shams Univ., 120 p.

Tarney, J., Saunders, A. D., Mattey, D. P. and Wood, D. A. (1981): Geochemical aspects of back-arc spreading in the Scotia Sea and western Pacific. Phil. Trans. R. Soc. Lond., A300, 263-285.

Tian, L., Castillo, P. R., Hilton, D. R., Hawkins, J. W., Hanan, B. B. and Pietruszka, A. J. (2011): Major and trace element and Sr-Nd isotope signatures of the northern Lau Basin lavas: Implications for the composition and dynamics of the back-arc basin mantle, J. Geophys. Res., 116, B11201, doi:10.1029/2011JB008791.

Vallier, T. L., Jenner, G. A., Frey, F. A., Gill, G. B., Davis, A. S., Volpe, A. M., Hawkins, J. W., Morris, J. D., Cawood, P. A., Morton, J. L., Scholl, D. W., Rautenschlein, M., White, W. M., Williams, R.W., Stevenson, A. J. and White, L. D. (1991): Subalkaline andesite from Valu Fa Ridge, a back-arc spreading center in southern Lau Basin: petrogenesis, comparative chemistry and tectonic implications. Chem. Geol., 91, 227-256.

Vermeesch, P. (2006): Tectonic discrimination diagrams revisited. Tectonic discrimination diagrams revisited, Geochem. Geophys. Geosyst., 7, Q06017, doi:10.1029/2005GC001092.

Wassef, B., Kamel, O. A., Armanious, L. K. and Sabet, A. H. (1973): Report on the results of the prospecting work for gold in El Sid-Semna area in 1970/1971. Geol. Surv. Egypt, internal report no. 23/1973.

Wilson, M. (1989): Igneous petrogenesis - A global tectonic approach. Unwin Hyman Ltd., 466p.

Zimmer, M., Kröner, A., Jochum, K. P., Reischmann, T. and Todt, W. (1995): The Gabal Gerf complex: A Precambrian N-MORB ophiolite in the Nubian Shield, NE Africa. Chem. Geol., 123, 29-51. 
بترولوجية وجيوكيميائية البازلت المتحول بوادى عطالله المُر، الصحراء الشرقية، مصر : بركنه فى حوض خلف قوس ناس ناضج نيويروتيروزوى

أيمن البلرى موريس

قسم الجيولوجيا - كلية العلوم - جامعة حلوان

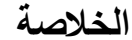

تتتنل الصخور البركانية المافية بوادى عطالله المر ، الصحراء الثرقية، مصر ، على صخور بازلت بورفيرى وغير

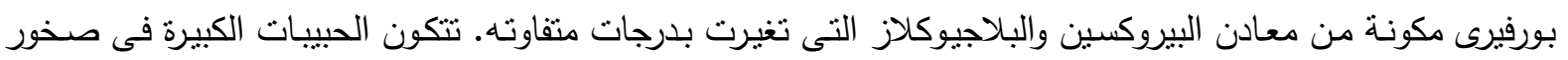

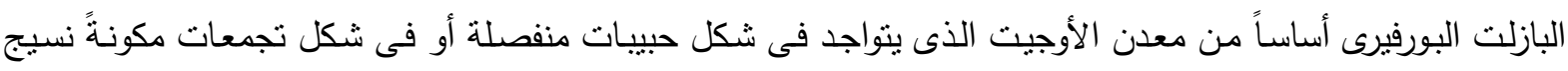

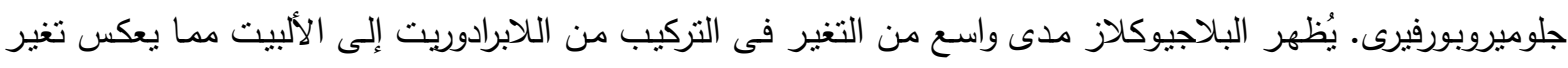

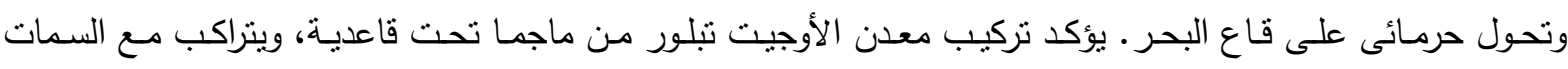

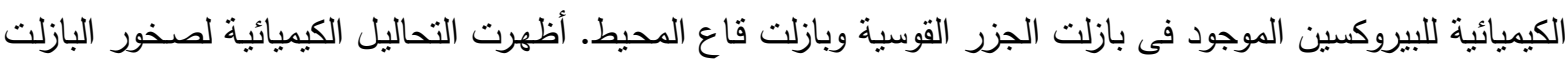

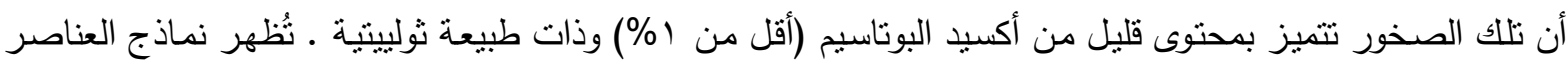

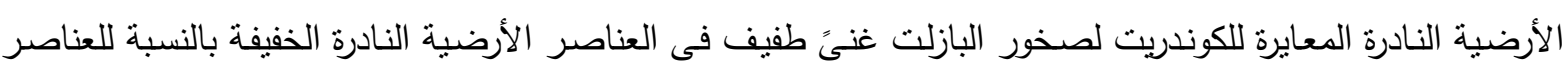

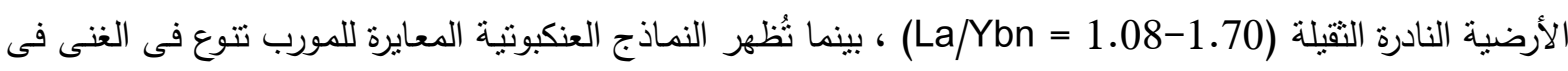

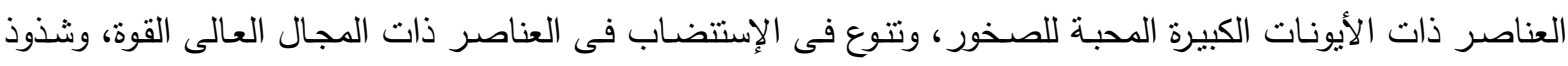

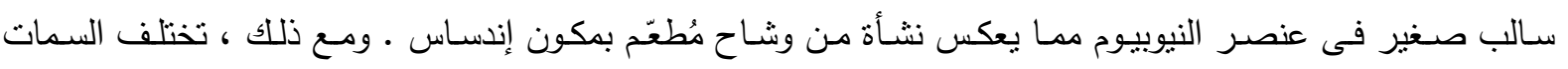

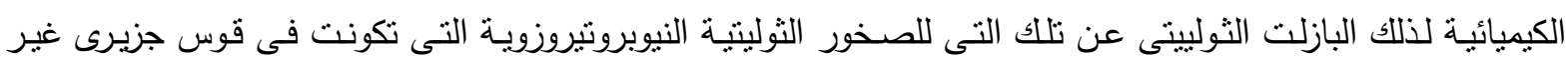

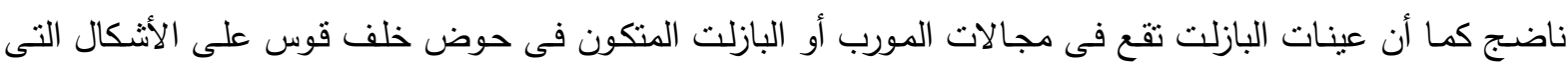

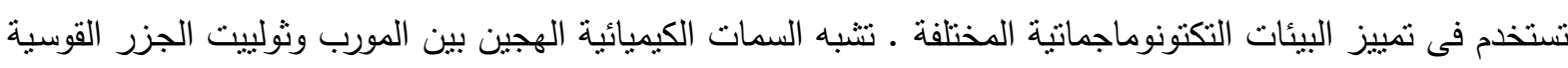

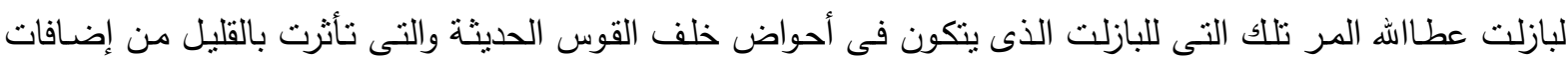

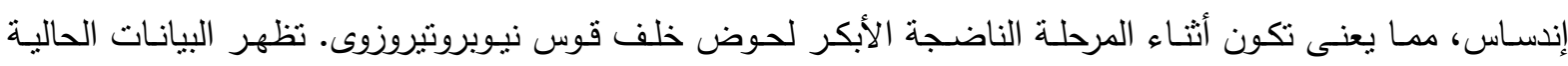

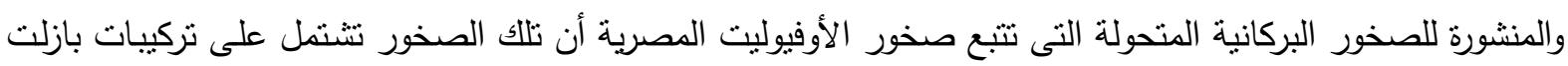
حوض خلف القوس والمورب • إن معظم صخور بركانيات الأوفيوليت المتحولة بمصر قد تكونت في بيئات تكتونيـة مرتبطة بالإندساس من وشاح أُغنى بمكون الإندساس بدرجات متفاوتة من زهيدة إلى كبيرة ، مما يعكس نشأة أثناء مراحل

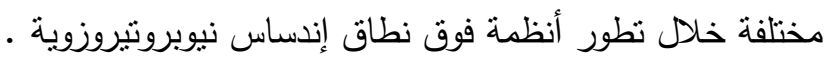


Maurice, A. E.

بترولوجية وجيوكيميائية سرينتين أوفيوليتى بمنطقة أم عش، الصحراء الثرقية، مصر : وشاح نيويروتيروزوى من

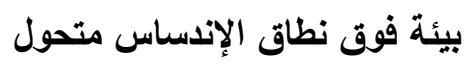

أيمن البلرى موريس الإنساس

قسم الجيولوجيا - كلية العلوم - جامعة حلوان

الخلاصة

يشتمل السربنتين الأوفيوليتى النيوبروتيروزوى لهنطقة أم عش بوسط الصحراء الثرقية الدصرية على أنواع كتلية

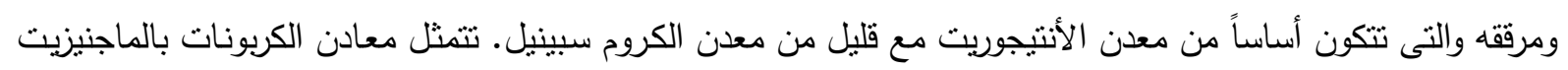

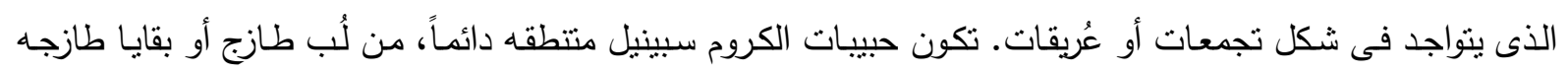

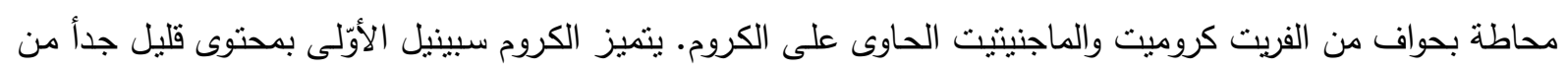

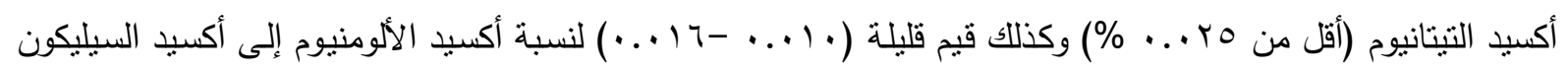

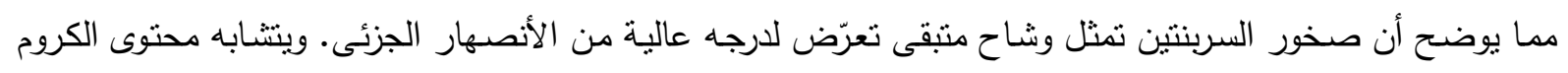

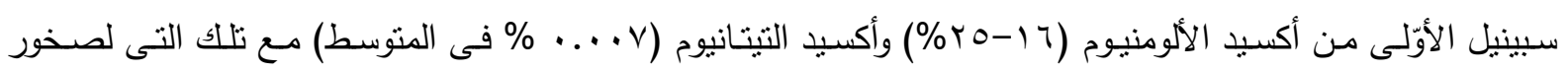

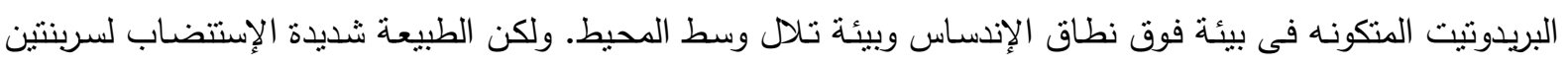

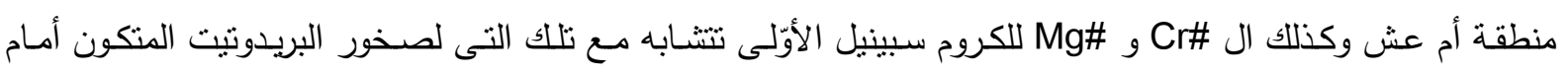
القوس لبيئة فوق نطاق الإندساس.

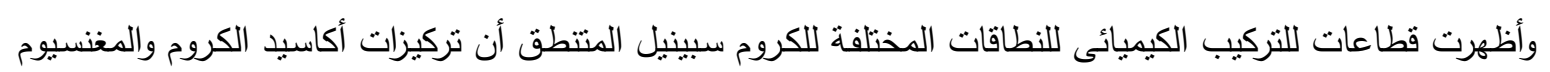

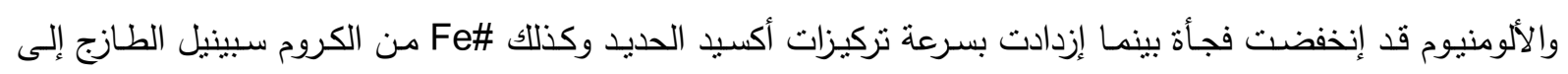

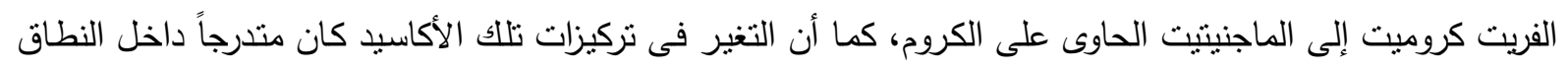

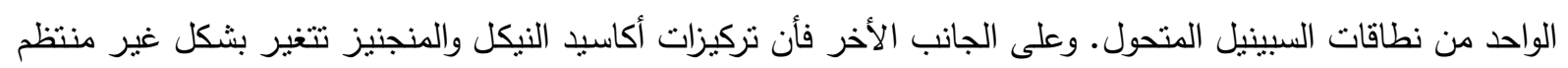

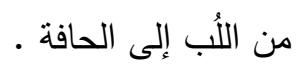

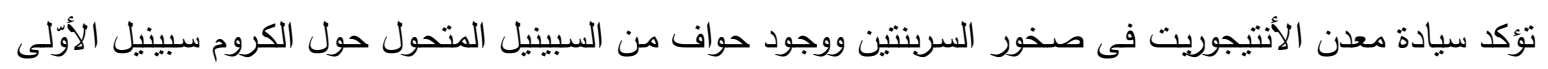

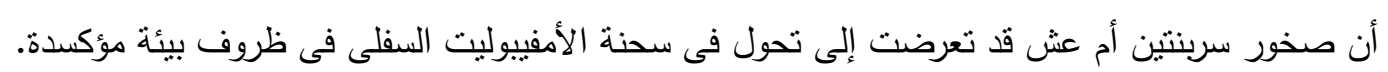


